\title{
La nascita del riso nella tradizione śivaita del Bengala medievale: un'epopea di frontiera
}

The Birth of Rice in Middle Bengali Śaivism: a Frontier Epic

\section{Fabrizio Ferrari}

\section{(2) OpenEdition}

\section{Journals}

\section{Edizione digitale}

URL: https://journals.openedition.org/mythos/3520

DOI: $10.4000 /$ mythos.3520

ISSN: 2037-7746

\section{Editore}

Salvatore Sciascia Editore

\section{Notizia bibliografica digitale}

Fabrizio Ferrari, «La nascita del riso nella tradizione śivaita del Bengala medievale: un'epopea di frontiera», Mythos [Online], 15 | 2021, online dal 15 décembre 2021, consultato il 16 décembre 2021. URL: http://journals.openedition.org/mythos/3520 ; DOI: https://doi.org/10.4000/mythos.3520

Questo documento è stato generato automaticamente il 16 décembre 2021. 


\section{La nascita del riso nella tradizione śivaita del Bengala medievale: un'epopea di frontiera}

The Birth of Rice in Middle Bengali Śaivism: a Frontier Epic

Fabrizio Ferrari

\section{L'agricoltura in India}

1 Le tribù vediche che nella seconda metà del II millennio a.C. giunsero nell'India nordoccidentale praticavano una forma di agricoltura essenziale limitata ai periodi di stanzialità (kșéma) che si intervallavano alla transumanza o ai raid guerrieri (yóga). Il riso (vrïhí; Oryza sativa L.), destinato a divenire l'alimento base in tutto il subcontinente indiano, non è attestato nella Rgvedasaṃhitā, fatto che ci permette di concludere che questa coltura non fosse nota nel periodo antico. Con lo spostamento dei centri di potere dal Pañjāb alla piana di Kurukșetra intorno al 1000 a.C., i clan vedici, allora organizzati in grandi confederazioni tribali (mahäjanapada), passano ad un'economia agricola $^{1}$. La cultura del riso diventerà infine dominante con il progressivo estendersi verso oriente della frontiera vedica e con l'inclusione nell'Āryavarta dei territori adiacenti al golfo del Bengala entro i primi secoli d.C. ${ }^{2}$.

2 Proprio nel Bengala, un'area tradizionalmente associata alla produzione di riso, come dimostrato dall'iscrizione di Mahāsthā (Baguṛā Distt., Bangladesh), probabilmente la più antica rinvenuta nel nord-est (III-II secolo a.C.) ${ }^{3}$, nascerà una tradizione letteraria dedicata alle origini mitologiche, alla produzione e alla lavorazione del riso ${ }^{4}$. Le fonti più importanti sono:

3 i. Dāker bacan e Khanār bacan, una serie di proverbi sul folklore contadino e la meteorologia, il cui nucleo è stato probabilmente composto tra l'viII e il xII.

4 ii. Śūnyapurān e Dharmapūjābidhān, entrambi attribuiti a Rāmāi Paṇḍit, il primo officiante del culto di Dharmarāj. In entrambe i testi troviamo due versioni di un 
cășāpālā, una sezione dedicata all'agricoltura (cāss) in cui sono narrate le vicende dello Śiva contadino (krșak Śib) ${ }^{5}$.

iii. La letteratura Śibāyan (xv-XVIII secolo), un filone poetico che celebra le vicende dello Śiva agreste. I testi di maggiore rilievo sono lo Śibāyan di Rām'kṛṣna Kabicandra (XVII secolo) e il Śib'samikìrtan di Rāmeśbar Bhațțācārya (inizio del xvIII secolo)' .

6 Nelle pagine a seguire ci occuperemo principalmente dei materiali attribuiti a Rāmāi Pandit in quanto, nella loro forma originale, rappresentano il prototipo di un genere divenuto di grande popolarità.

\section{II mito della nascita del riso}

7 Lo Śūnyapurāṇ (ŚP) è un testo in medio-bengali attribuito a Rāmāi Paṇḍit ${ }^{7}$ che nella sua forma attuale risale ad un periodo non precedente il XV secolo. ${ }^{8}$ Redatto principalmente nei metri paýär e tripadi ${ }^{9}$ si presenta come un lavoro eterogeneo che include narrative mitologiche e istruzioni rituali per la celebrazione del Gājan, il festival annuale dedicato a Dharmarāj/Dharma Ṭhākur. Questi è una divinità solare legata ai culti della fertilità di Rậrh, un'antica realtà geopolitica del Bengala occidentale divisa dal fiume Ajaỷ in Uttar Raārh e Dakṣin Rạaṛh e corrispondente all'incirca ai distretti centrali del West Bengal: Birbhum, Barddhaman, Bankura, Hooghly, Howrah, Medinipur e Murshidabad (Fig. 1).

Fig.1. Mappa politica del West Bengal (India).

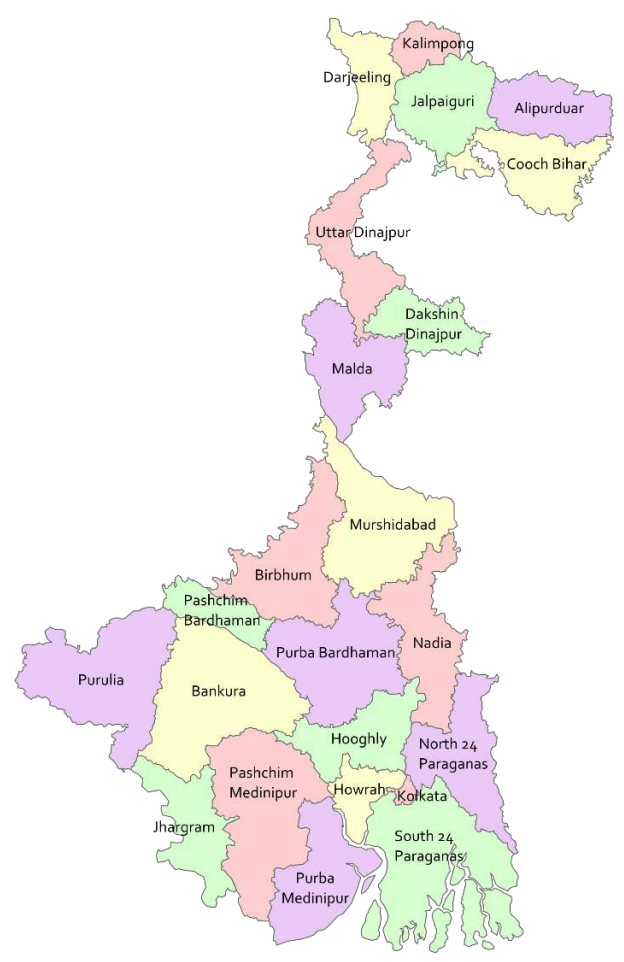

Wikimedia

8 Il Gäjan di Dharma (Dharmer gājan), oggi una tradizione quasi completamente assorbita dallo śivaismo bengalese, si svolge alla fine di Caitra (marzo/aprile), l'ultimo mese del calendario bengali, ed era in origine una festività legata a caste ritenute "inferiori" 
(antyaja) quali d̦om, bāgdi e hāri. Sebbene nel Bengala orientale sia attestata una forma di Gājan dedicata a Śiva (Śiber gājan) e nota come Nî̀l'pūja $\bar{a}^{10}$, è generalmente accettato che il culto di Dharmarāj ne rappresenti la forma originale. Sulla problematicità di questa opinione ritorneremo oltre.

L'aspetto distintivo del Gājan è costituito da una serie di pratiche di mortificazione che evocano le gesta di Lāusen, l'eroe dell'epica popolare di Rāạh narrata nei Dharmamangal '-kābya. Dopo un rito di iniziazione in cui i devoti consacrati (bhaktya) divengono rinuncianti (sannyāsī), questi prendono parte a una serie di rituali che includono il lanciarsi su letti irti di spine o paletti acuminati (pātt), il forarsi la lingua e gli arti superiori con piccoli tridenti e, infine, la celebre Carak pūja $\bar{a}$ (Fig. 2), nota in inglese come hook swinging. Il rito prevede che il devoto si faccia trapassare i muscoli dorsali con grossi uncini collegati con delle funi ad una piattaforma sospesa e che, così assicurato, oscilli avanti e indietro ${ }^{11}$ in taluni casi lanciando fiori, riso o reggendo infanti ${ }^{12}$.

Fig. 2. Celebrazione della Carak pūjā durante il Gājan.

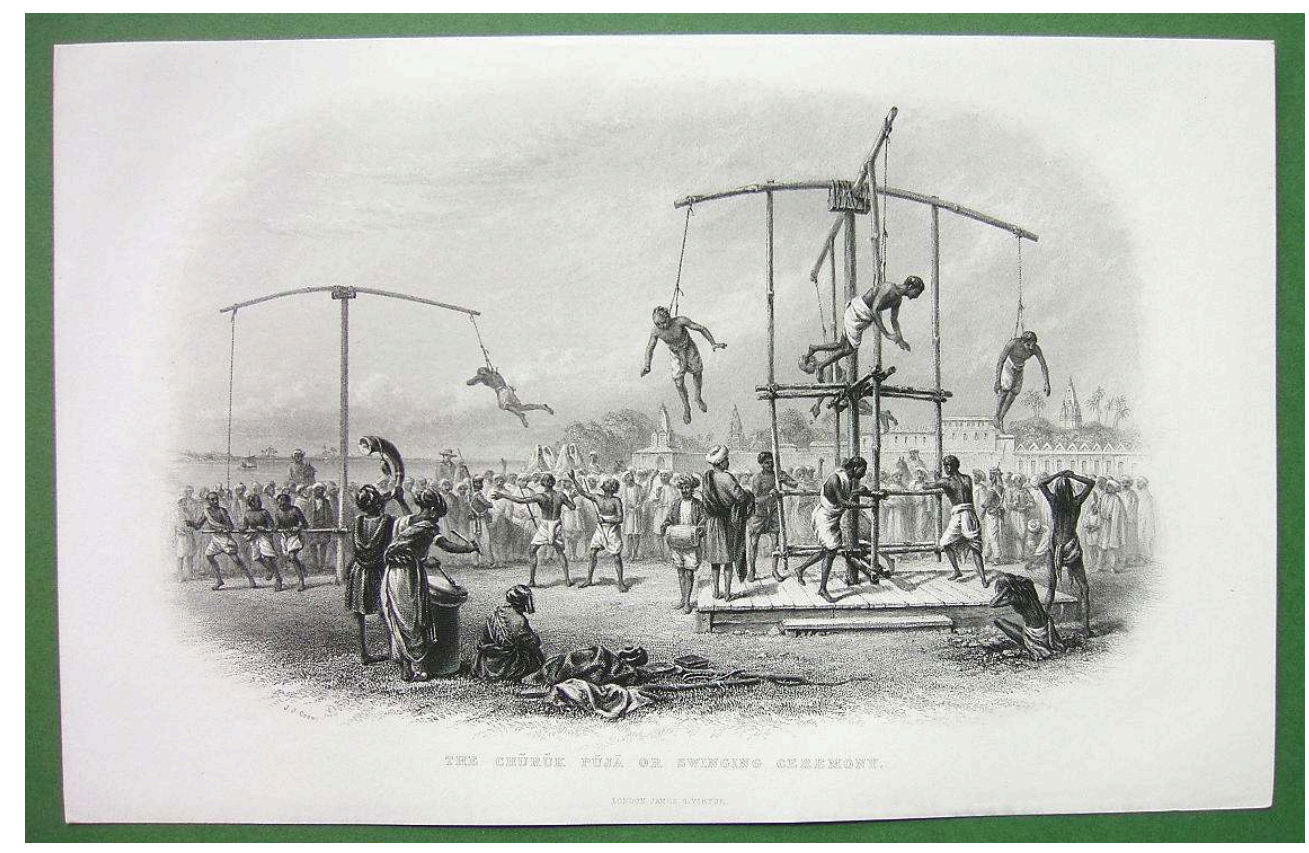

NoLAN 1859, 471

Tali pratiche sono menzionate per la prima volta nel Candìmañgal di «Kabikaṅkan» Mukundarām Cakrabartī, un poema composto nel 1589:

caitramāse pūje śib nānā upacāre /

ḍāk ḍol bādya bāñge śiber mandire /

jihbā phơre jihbā kāțe karaỷe carak /

abhimata sbarge yāỳ nā yāỳ narak //13

Nel mese di Caitra [i devoti] celebravano Śiva con molte offerte.

Nel tempio di Śiva risuonavano invocazioni e il suono di grandi tamburi.

Si bucavano e si tagliavano la lingua e praticavano la Carak[püjā $]$.

Raggiungevano così il paradiso di loro scelta, e non l'inferno.

Mukundarām, in quanto nativo di Barddhaman e attivo a Medinipur, è culturalmente legato a Rạạhh, dove il culto di Dharmarāj era maggiormente diffuso. Il fatto che questi 
menzioni Dharmarāj ma si riferisca alla Carak pūjā come a un rito dedicato a Śiva, farebbe pensare che già nel XVı secolo l'elemento śaiva fosse dominante ${ }^{14}$.

In ŚP, l'unica sezione in cui Śiva ha un suo ruolo indipendente, per quanto gerarchicamente subordinato a Dharma Ṭhākur, è il mito della nascita del riso ${ }^{15}$. Egli è qui ritratto come un dio agreste ( $k r s ̦ a k$ debatā) le cui vicende offrono uno spunto per comprendere la realtà e la ricchezza della cultura contadina del Bengala medievale.

13 Śiva è inizialmente rappresentato come un individuo ai margini ${ }^{16}$ : un povero mendicante, ${ }^{17}$ spesso avanti negli anni (bura), incapace di mantenere la propria famiglia per via della propria dipendenza da gañjā (Cannabis sativa L.) e dhustur (Datura metel L.):

3. jakhan āchen gosāñi haā digambar / ghare ghare bhikhā māgiā bulen ìsar //

4. rajanī parabhāte bhikkhār lāgi jāi / kuthāe pāi kuthāe na pāi //

5. hattukī baeṛā tāhe kari dinpāt / kata haras gosāñi bhikkhāe bhāt //

6. āmhar bacane gosāñi tumhi cas cās / kakhan anna hae gosāñi kakhan upabās //18

3. Quando il Signore [Śiva] era un asceta nudo, andava di porta in porta a chiedere l'elemosina e diceva:

4. «Chiedo la carità [fin] dalla mattina presto. In alcuni posti, mi danno qualcosa, in altri niente.

5. [E allora] passo la giornata [cibandomi] di hattukī e beherāa ${ }^{19}{ }^{»}$. Come era contento il Signore con il cibo ricavato dalla questua!

6. [Ma] segui il mio consiglio, o Signore: passa all'agricoltura, [perché] a volte c'è da mangiare, ma a volte devi digiunare.

Esausta dalla propria situazione, Pārvatī, moglie di Śiva, cerca in ogni modo di spronare il marito ad assumersi le proprie responsabilità e sostenere la famiglia:

9. ghare dhānna thākilek prabhu sukhe anna khāba / annar bihane prabhu kata dukha pāba //

10. kāpāsa casaha prabhu pariba kāpaṛa / kata nā pariba gõsāi keodā bāghar chara///

11. tila sarisā cās kara gõsāi bali taba pāe / kata nā mākhiba gosāñi bibhūti gulā gāe// 20

9. «Marito mio: se in casa c'è il riso, con quanto piacere lo mangeremo! Ma senza riso, soffriremo tutti terribilmente!»

10. «O mio signore, coltiva il cotone, così che possiamo avere vestiti! Quanto ancora credi di poter indossare una pelle di tigre?»

11. «Ti prego, marito: coltiva sesamo e senape. Per quanto ancora credi di poterti coprire il corpo con la cenere?»

Si noti come, ai vv. 12-13, Pārvatī segnali come i frutti del lavoro nei campi siano necessari non solo per la mera sopravvivenza ma anche per poter presentare le necessarie offerte rituali a Dharmarāj:

12. mug bāțalā āra casin ikhu cāsa / tabe habeka gõsāi pañcāmartara āsa //

13. sakal cās cas prabhu āra ruio kalā / sakal dabba pāi jena dhammapūjār belā //21

12. "Coltiva fagioli verdi, ceci e canna da zucchero. Allora avremo il piacere di offrire pañcāmrta ${ }^{22}$ per propiziare il Signore [= Dharmarāj].»

13. «Coltiva tutti i tipi di piante, o Signore, e pianta alberi di banane. Ci servono tutte le offerte possibili per Dharma[rāj].» 
16 dedicarsi al lavoro nei campi. Crea allora i due agricoltori (hele) - Man («Mente») e Paban («Vento») - e un aratro (längal) d'oro munito di un coltro (phāl) d'argento. La descrizione del lavoro è di grande realismo: Śiva applica una coppia di doghe (sali) al giogo (joyal) e assicura un'imbragatura (joti) in modo da poter mantenere un fermo controllo sulle maniglie (āngad) dell'aratro e quindi orientare il timone (pācan bāri). Infine, appronta un erpice (mai) i cui lati sono assicurati con due corde (dari).

In alcune varianti del mito, come la versione che troviamo nel più tardo Śib'sanikirtan di Rāmeśbar Bhațțācārya, la giovane moglie di Śiva invita il marito a trovare un lavoro stabile e smettere di fare il mendicante. Śiva sembra essere d'accordo ma non ha le finanze necessarie (sampatti) per un'attività in proprio (bānijya). Inoltre, essendo un dio, non può certo lavorare per gli uomini. L'agricoltura è un compromesso accettabile perché gli permetterebbe di lavorare con altre divinità ${ }^{23}$. Viśvakarma, artigiano degli dèi, forgia quindi un aratro dal tridente di Śiva. Indra, re degli dèi, gli affitta un campo ma per pagare la tassa, così come per l'acquisto delle sementi e degli attrezzi agricoli, Śiva è costretto a rivolgersi a Kubera, signore degli yakșa e delle ricchezze, qui ritratto come un usuraio. Infine, Yama, dio della morte, presta a Śiva il proprio bufalo per tirare l'aratro ${ }^{24}$. In ŚP, invece, Viśvakarma, prepara cento falcetti d'oro che saranno poi utilizzati dal toro Nandi per la mietitura. L'eroe epico Bhīma viene quindi invocato per fare da coordinatore (moralgiri) del lavoro dei contadini mentre Hanumān svolge il compito di «protettore dei campi» $(k o t ̦ a \bar{l})^{25}$.

A questo punto, Rāmāi illustra il susseguirsi dei vari lavori, dalla fertilizzazione dei campi alla semina fino al raccolto ${ }^{26}$. Nel mese di Māgh (gennaio/febbraio), Hara (=Śiva) prepara la terra che dovrà ricevere i semi (dhan bihan/bic dhān). Pārvatī, nel frattempo, inizia a sentire la lontananza del marito e ne chiede il ritorno sul monte Kailaś. Una volta raggiunta la consorte, cominciano una serie di effusioni e giochi erotici, qui parzialmente sottointesi, fino al punto in cui Śiva non riesce più a trattenere il proprio amore. Dall'emissione del proprio desiderio erotico $(k \bar{a} m)$ [=liquido seminale], nasce il riso primordiale detto kāmad («desiderio»):

22. ek din ras hāse kailāse bholānāthe /

pem rase tilocan pābbatīr māthe //

23. kautuk karite sibe upajila kām /

kāme upajila dhān kāmada bali nām //27

22. Un giorno, sul monte Kailaśa, Bolānāth [=Śiva] sorrise.

Trilocana ${ }^{28}$ [guardò] con desiderio il volto di Pārvatī.

23. Immerso nei giochi [erotici], il desiderio di amore di Śiva crebbe

e da tale desiderio nacque il riso kāmada.

19

Il testo diviene qui estremamente ripetitivo ma di grande interesse per lo storico dell'agricoltura e il botanico. Rāmāi elenca infatti i nomi di tutte le specie di riso nate dalla varietà primordiale (eha dhāne haibāk sahasrek nām), offrendo così uno spaccato unico della cultura cerealicola nel Bengala medievale ${ }^{29}$ : 


\begin{tabular}{|c|c|c|c|}
\hline$p \bar{a} d$ & riso & $\begin{array}{l}p \bar{a} d \\
23 \mathrm{~b} \\
56 \mathrm{~b}\end{array}$ & $\begin{array}{l}\text { riso } \\
\text { kāmad } \\
\text { muktāhār }\end{array}$ \\
\hline $57 a$ & jețh dhān; chicharā; āmla & $57 \mathrm{~b}$ & ālācit*; phepheri \\
\hline $58 \mathrm{a}$ & sanā kharaki*; duggābhog; āsaāñga; kala & $58 \mathrm{~b}$ & miktāhār \\
\hline $59 a$ & kālā mugar & $59 b$ & nāgar juān* \\
\hline $60 \mathrm{a}$ & tulā sāli & $60 \mathrm{~b}$ & $\overline{a s a t i}$ \\
\hline $61 a$ & bak kari & $61 b$ & gotam palāl; pātal \\
\hline $62 \mathrm{a}$ & pāngusisiā; bhāddamukhi; khemrāa* & $62 \mathrm{~b}$ & tulān dhān; dudurāa \\
\hline $63 a$ & gujurā; boāli; ḍ̂ār; hāti pāñjar & $63 \mathrm{~b}$ & bựā māttā \\
\hline $64 a$ & til sāgari & & \\
\hline $65 a$ & latāmou; moukalas; khejur chaṛi & $65 \mathrm{~b}$ & pabbat jirāä; gandhatulsī; dalā guṛi \\
\hline $66 a$ & bandhi; bã̄s gajā; sītāsālì & $66 \mathrm{~b}$ & $\begin{array}{l}\text { hukuli; harikāli*; kusum māli (= } \\
\text { sâli) }\end{array}$ \\
\hline $67 a$ & raktasāl; candan sāl & $67 \mathrm{~b}$ & rāj dal; moukalas \\
\hline $68 a$ & ựāsālī; bindhasali; lāıśālī & $68 \mathrm{~b}$ & bhādolì \\
\hline $69 \mathrm{a}$ & rājdal; moukalas; ājān siali (= śāli) & $69 b$ & kālā kāttik; megi \\
\hline $70 \mathrm{a}$ & $k h \bar{r}$ kamb $\bar{a} ;$ ranajan $a$ & $70 \mathrm{~b}$ & kāmad \\
\hline $71 a$ & (khudda) dudurāa; bhajanā; bã $k$ ai & $71 b$ & mūlāmuktahār \\
\hline $72 \mathrm{a}$ & 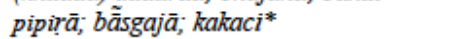 & $72 b$ & mādhabalatā; bāgan bici \\
\hline $73 a$ & koțā; rāagar; tojana $\bar{a}^{*} ;$ bora & $73 b$ & $\begin{array}{l}\text { koñgar bhog; jalā rāngi*; } \\
\text { kanakcur }\end{array}$ \\
\hline $74 a$ & lälkāminī; solpana $\bar{a}^{*} ;$ pärcchā bhog & $74 b$ & āndhārkuli; gopālbhog \\
\hline $75 a$ & bukhi; ājōn lakkhī; bā̄smatī & $75 b$ & sāl chātīi; kāngad; gandhamālatī \\
\hline $76 a$ & $\bar{a} m$ pāban*; gā̄ bāli; pātharāa & $76 \mathrm{~b}$ & masi loț; jhing $\bar{a}$ sâl; tasara $\bar{a}^{*}$ \\
\hline $77 a$ & sam dhumā; suā sān; țāngan & $77 b$ & hari; mahīpāl; b äkssāl \\
\hline
\end{tabular}

20 Tra Āṣạ̣̄h (giugno/luglio) e Bhādra (agosto/settembre), i semi vengono piantati e sono descritti nella loro lenta maturazione. A questo punto, Rāmāi fa riferimento a due tecniche: (i) la coltivazione del riso in asciutta, preceduta dalla pratica del debbio (jhum), che troviamo nelle zone collinose e pedemontane (dāngar); e (ii) le caratteristiche risaie sommerse, tipiche delle piane alluvionali (dahar).

21 La tecnica del debbio, un incendio controllato della biomassa, è spesso legata ad un'agricoltura di sussistenza e fu praticata fino a tutto il xviII secolo nell'India nordorientale (Arunachal Pradesh, Meghalaya, Mizoram e Nagaland) e nei distretti settentrionali del Bangladesh (Rangamati, Khagrachari, Bandarban e Sylhet):

This appellation of "juming" is given to the system of cultivation which is universally followed by all hill tribes in India, from the Himalayas to the furthest point of Siam. A suitable spot is selected, by choice on the breezy shoulder of some hill; the undergrowth is cut and the smaller trees felled, the large forest trees being left standing, on account of the labour required to cut them down. A spot covered with a growth of bamboo is considered the best, as the bamboo ash is a good fertiliser, and the ground is easily cleared. When all is cut, the mass is left to dry in the sun, and then heaped up and fired. The villagers stand round the clearing with long bamboo sticks to beat the flames and prevent them spreading to the forest. But if a high wind comes, their efforts are occasionally vain, and then is to be seen that grandest and most awful of all spectacles-a forest fire ${ }^{30}$. (Asiatic Society of Calcutta), copiato nel 1117 Bs (1710/11 d.C.) da un certo Arjun Paṇdit, figlio di Daỷāāām Paṇdit:

kāṭila sakal dhānya halya āḍāi hālā /

kṣetre rāthi brọkodar śibpāśe gelā //

bhīme dekhi śankar jijñāse haŷyā druta /

kaha kaha brokodar dhānya hala kata //

eta śuni brọkodar sadāśibe bale / 
ārāi hālā dhānya mātra haila sakale //

kruddha haẏyā śib bale cāser kibā gun /

maruk mane cās karmme lāguk āgun //

eta śuni agni jālyā dila brọodar /

puḍite nāgila dhānya dbādaś batsar $/ /{ }^{31}$

[Bhīma] raccolse il riso: due hālā e mezzo in totale. ${ }^{32}$

Pancia-di-Lupo [=Bhìma] lo dispose sul campo e andò da Śiva.

Vedendo Bhīma, Śiva subito chiese:

«Dimmi, dimmi: quanto riso abbiamo fatto?»

Bhīma ascoltò e [così] rispose a Sadāśiva:

«In totale solo due hālā e mezzo.»

Śiva si infuriò e disse: «Quale sarebbe il vantaggio di fare il contadino?

Che l'agricoltura sia dannata! Brucia tutto!»" ${ }^{33}$

Ricevuto l'ordine, Pancia-di-Lupo incendiò [il campo].

Il riso bruciò per dodici anni.

Il secondo è estrapolato dall'edizione Basu, ricavata dal MS G5424 (Asiatic Society of Calcutta). Anche in questo caso, Bhīma riceve l'ordine da Śiva di bruciare il raccolto fino a quando, su invito della dea, vengono chiamate a raccolta tutte le divinità:

53. indar baliā hara pārila hũkār /

chisți rakkhā kara indar haila chārkhār //

54. khir kuṇdar khir amarta kuṇ̣ar pāni /

amarta barisan indar karila āpuni //

55. gosāñi dilen tabe biunir bāa /

jata chila chār pãs ựiāt jāa //

56. punarapi gosāñi chihattha bulāila /

jemati dhān chila pūrbba temati haila //34

53. Hara invocò Indra a gran voce [e] gli chiese di salvare il mondo dalla catastrofe.

54. Indra stesso fece precipitare una pioggia divina di acqua e latte dalle riserve celesti.

55. Il Signore [Śiva] si mise a fare aria con un ventaglio fatto di canne di bambù [così che] tutta la cenere e i detriti [da combustione] fossero rimossi.

56. [Quindi] il Signore toccò nuovamente [la terra] con mano propizia [e] il riso spuntò come e quanto prima [del fuoco].

Soprattutto nel secondo passaggio, Rāmāi descrive la pericolosità del debbio, una pratica che presenta rischi non indifferenti, come evidenziato nella testimonianza del capitano Thomas E. Lewin, Deputy Commissioner of the Chittagong Hill Tracts District (oggi in Bangladesh):

The firing of the jooms is sometimes a source of danger, as at that season of the year the whole of the surrounding jungle is as dry as tinder, and easily catches fire. In this way sometimes whole villages are destroyed, and people have lost their lives. I have myself seen a whole mountain-side on fire for four days and four nights, having been ignited by joom-firing. It was a magnificent sight, but such a fire must cause incalculable injury to the forest..$^{35}$

La seconda tecnica di coltivazione di cui troviamo traccia in ŚP è quella delle risaie sommerse. Śiva viene descritto mentre raccoglie dalle rive di uno stagno il limo (pali) con cui nutre il terreno di coltura che poi irrigherà. A quel punto le plantule (bicchān) vengono trapiantate nei campi allagati dai numerosi effluenti del fiume che nel testo è 
chiamato Gañgā (Gange) ${ }^{36}$ ma che proponiamo di identificare con la Burigañgā («Vecchio Gange»), un fiume che scorre a sudovest di Dhaka.

Nel mese di Āśvin (settembre/ottobre), le piogge continuano a bagnare la terra assicurando nutrimento alle piante che crescono e maturano. Il testo fa riferimento al «riso galleggiante» (jalidhān), una varietà di Oriza sativa L. che risponde alle continue inondazioni e può crescere fino a cinque metri. Infine, tra Kārtik (ottobre/novembre) e Agrahāỳạ (novembre/dicembre), le spighe (śiș) sono pronte per il raccolto: tagliate con degli appositi falcetti, vengono disposte in fasci (birāa). Segue una sezione di otto versi in laghu tripadī seguito da colophon intitolata ḍhenki mangal, in cui si celebra un attrezzo agricolo tipico della cultura contadina del Bengala il cui uso rituale è ancora oggi diffuso in quelle cerimonie legate al ciclo della terra e, più in generale, alla fertilità.

\section{La mondatura del riso ${ }^{37}$}

Il ḍhẽki ${ }^{38} \mathrm{è} \mathrm{un} \mathrm{attrezzo} \mathrm{usato} \mathrm{per} \mathrm{rimuovere} \mathrm{le} \mathrm{glumelle} \mathrm{o} \mathrm{altre} \mathrm{forme} \mathrm{di} \mathrm{rivestimento}$ dalle cariossidi del riso. Si tratta di uno strumento principalmente legato al lavoro femminile ${ }^{39}$. Consiste in una leva di legno lunga circa due metri, solitamente realizzata in legno di mango (ām) o giaca $(k \tilde{a} t a \bar{a} l)^{40}$, sospesa su di un perno tra due montanti verticali e bilanciata ad un'estremità con la pressione del piede e all'altra, montato ad angolo retto, da un corto pezzo di legno cilindrico (lungo circa $60 \mathrm{~cm}$ e di $20 \mathrm{~cm}$ di diametro) con la base ricoperta da una placca di ferro che funge da pestello. Quando la leva viene sollevata, la donna che la aziona toglie il piede in modo che la trave venga rilasciata e il pestello cada sui cereali ammassati su una base circolare sormontata da una spessa tavola di legno (Fig. 3-5 $)^{41}$.

Fig. 3. Donne vagliano il riso con ộẽēi (sinistra) e śūrpa (destra).

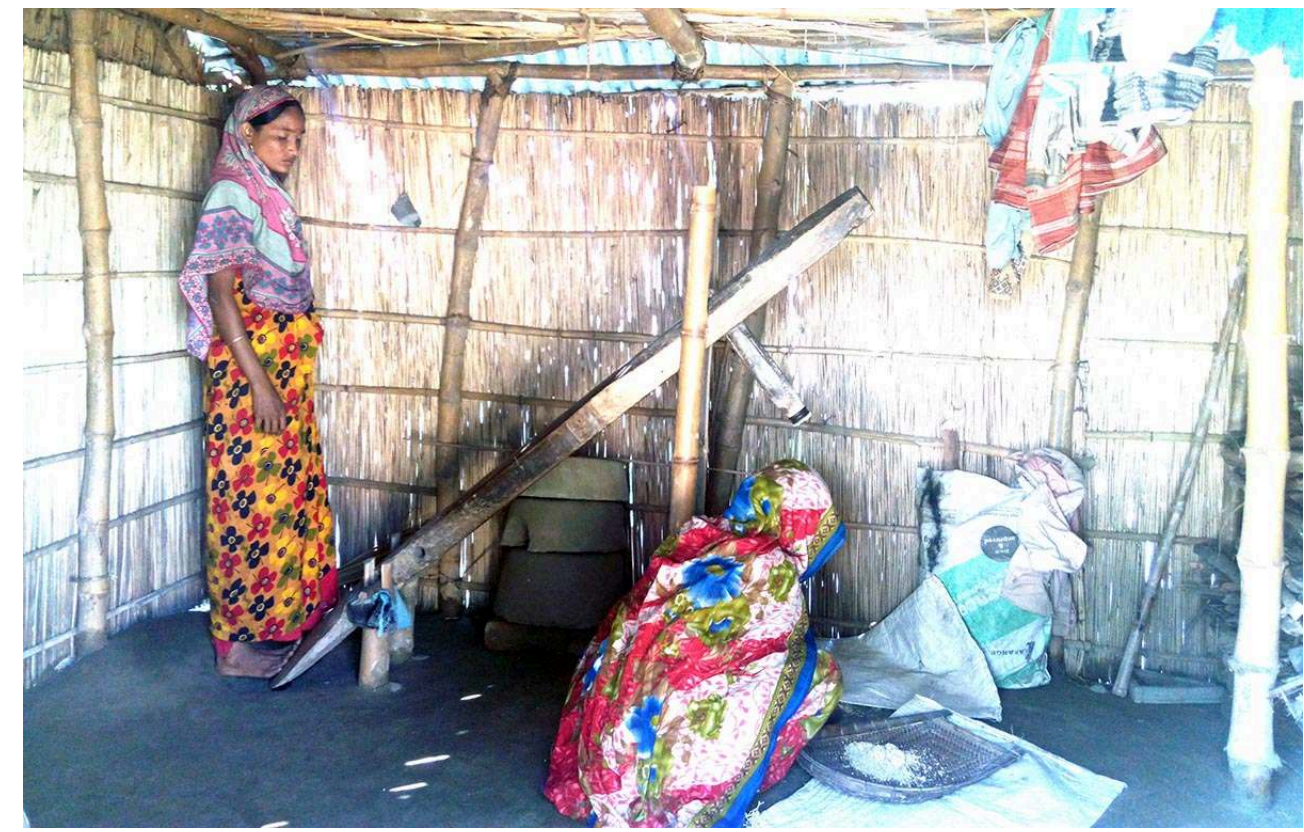

Deoȳāngañj, Jamalpur District, Mymensingh Division (Bangladesh), dicembre 2019.https:// banglarchithi.com 
Fig. 4. Donne al lavoro con il ventilabro (śūrpa) a sinistra e il ḍhẽkī, a destra.

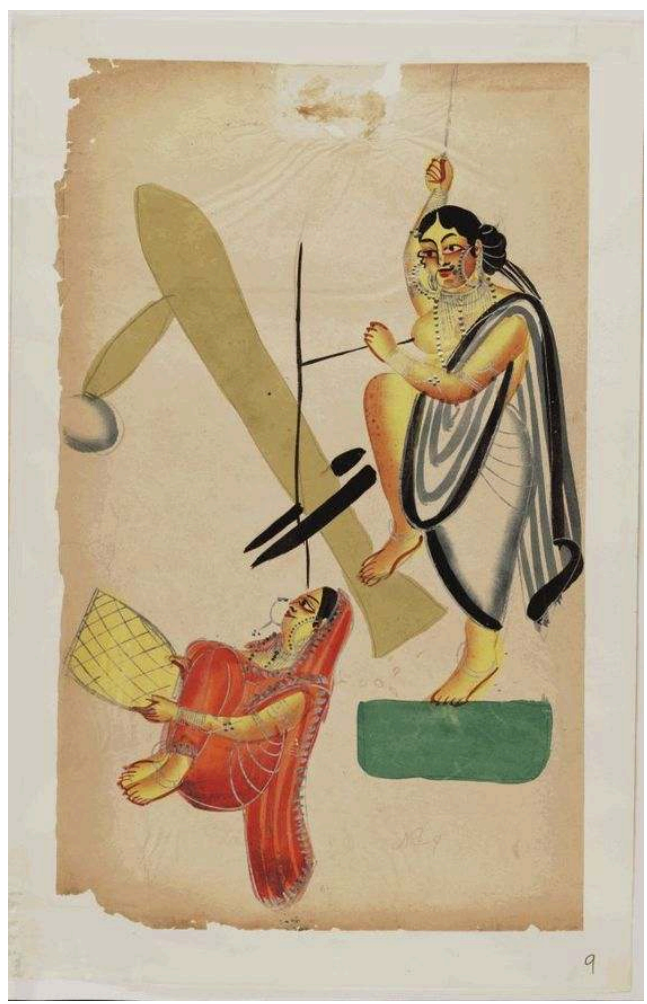

Acquerello su carta (pațacitra), xıx secolo, della scuola di Kālīghāt, Kolkata (WB)

Fig. 4. Donna intenta a vagliare il riso con il ḍhẽkī.

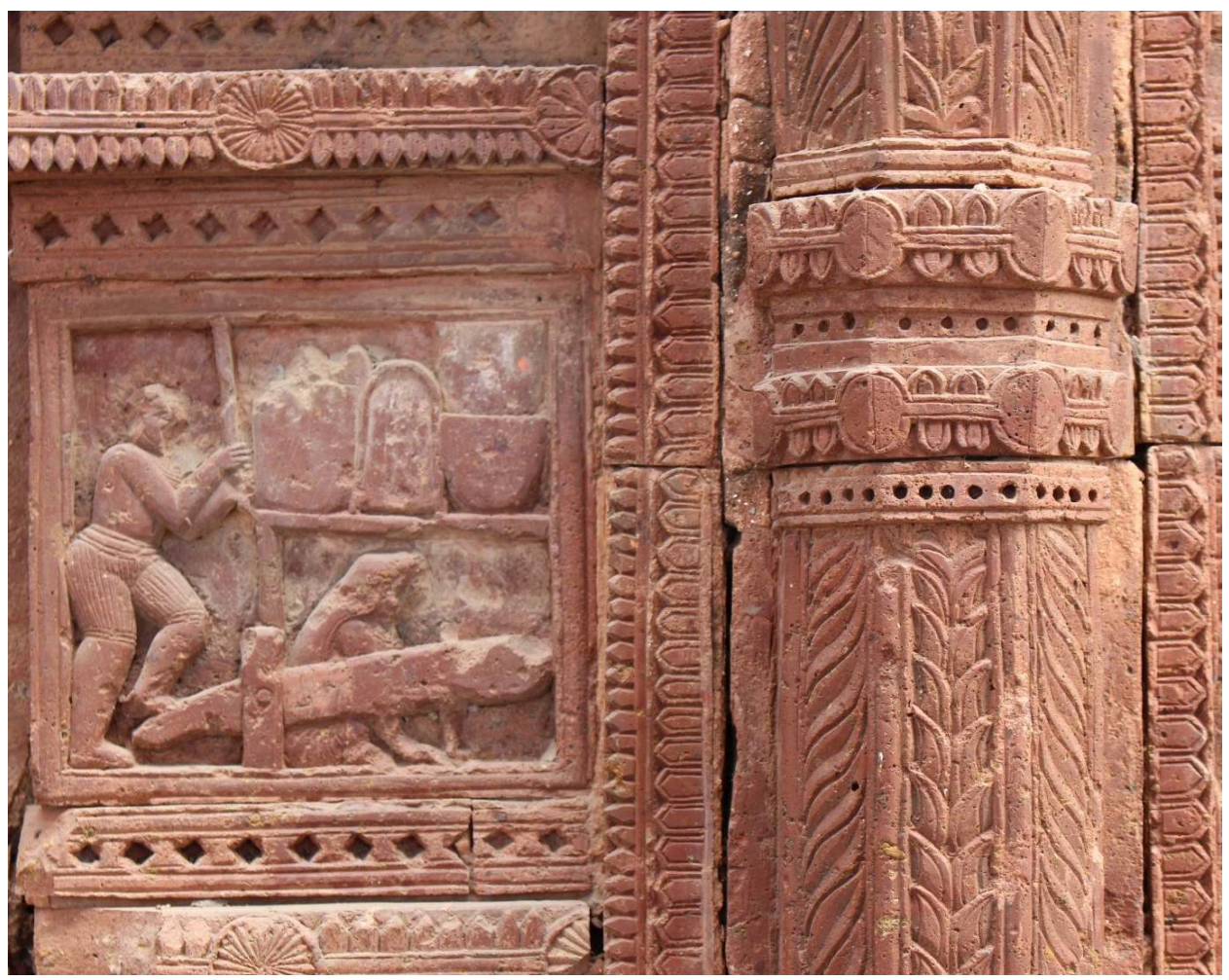

Complesso templare di Ganpur, Birbhum (WB), xvIII secolo 

usavano per la mondatura dei cereali mortaio e pestello, bastoni (danda) (KKS 563) e la forza di animali quali buoi e bufali, che venivano lasciati camminare su e giù per l'aia (KKS 487). Troviamo tuttavia un interessante riferimento ad un attrezzo simile al dhẽeki nella Piastra di Vișnusena, (VI-VII secolo d.C.), dove si parla di una macchina costruita come una leva per estrarre l'acqua da un pozzo ${ }^{43}$.

Al dhễkī, che è celebrato come il veicolo (bāhan) del saggio Nārada (Figg. 6-8), vengono tributate offerte di vario genere, canti e i tradizionali ululati (hulāhuli dhbani) di buon auspicio in occasioni quali matrimoni (bibāh), il primo boccone di riso di un infante (anna prāśan), la festa del raccolto (Nabānna Utsab, verso metà novembre) o durante il solstizio d'inverno (Pouṣ Samkrānt). Il palo che funge da leva è di solito spalmato con pasta di sandalo o decorato con disegni floreali. Su di esso vengono poggiate ghirlande fragranti, bastoncini di incenso e statuette di terracotta rappresentanti cavalli, elefanti, rane e tartarughe, tutti animali legati alla fertilità ${ }^{44}$. Il ḍhenkimangal che troviamo in ŚP riflette appieno questo tipo di pratiche:

1. kautuket deagan karite mangalan basilā bambhā bisțu har / tettis koți deb basilen sab gandharbba kinnar //

2. paṇdit cāri jane ānandit pūra mane dbādaś bhakta āmani / muktahār dhānna āni mukutā prabāla māni durlabha jagateta bākhāni //

3. koțāl cāri jane ādesi debgane nārade ānāha tarāgati / calila tatahpar muni barābar kahila debar bhāratī //

4. suniā munirāj bāhan karila sāj ḍ̣ẽki pițhe kari ārohan /

bhābi jugesar calila munibar suniā bāramati bharan //

5. tețhanga haiā jāa bhekar sangit gāa ựila deb biddamāne /

dekhiā debgan ādare tatakhan basāila ratnasimhāsane //

6. tideb mahārājā dệkīr karilā pūjā sugandhi puppar mālā diā /

debkannā meli diā hulāhuli ānandeta ḍhẽkī mañgaliā //

7. bājae jaeḍhāk meghar sam ḍāk sunite sudhani bājanā /

mṛdanga kārāā bāje phular mālā sāje ānandeta dharmmar pūjana //

8. paṇdite bedgān nichiā pelen pān hului parae ghane ghan /

sumadhur bājanā suni mukutā hār āmani ḍhẽkī e kara ārambhan //

1. Brahmā, Viṣnu e Hara sedettero insieme per preparare di comune accordo gli utensili per la cerimonia propiziatoria. I trentatré dèi si riunirono con tutti i gandharva e i kinnāra. ${ }^{45}$

2. Col cuore traboccante di gioia, dodici devoti [si rivolsero] così ai quattro pandit: ${ }^{46}$ «Portiamo il riso muktahāra, celebriamo i germogli di riso e cantiamo la durezza di questa vita».

3. Gli dèi ordinarono ai quattro guardiani ${ }^{47}$ di andare prontamente a chiamare Nārada. Questi raggiunsero il saggio e gli riferirono quanto detto dagli dèi.

4. Sentito [ciò], il signore dei sapienti approntò la sua cavalcatura e salì sul ḍhêkî. Anche Yugeśvara (=Dharmarāj) giunse quando udì che il sommo sapiente stava preparando le dodici offerte.

5. Accompagnato dal canto delle rane, [Nārada] volò sul tripode [d̂êeki] verso la dimora degli dèi..$^{48}$ Guardò con rispetto in direzione gli dèi e quindi si sedette sul trono.

6. Il Signore del Cielo presentò le offerte al ḍhêkī insieme ad una ghirlanda di fiori fragranti. Celesti damigelle lo ricevettero al suono di ululati e canti propiziatori.

7. Ascoltare il dolce suono dei canti è come sentire il tuono nelle nubi. Adorate Dharma[rāj] con letizia indossando ghirlande di fiori mentre risuonano [i tamburi] mridanga e kārā. 
8. I sacerdoti offrirono inni vedici [e] betel [mentre] si susseguivano in rapida successione ululati [di gioia]. Udendo i dolci canti, il ḍ̂êki immediatamente iniziò a pestare il riso.

Fig. 6. Nārada raggiunge Śiva e Pārvati, assisi sul toro, a cavallo del ḍ̂ẽēi.

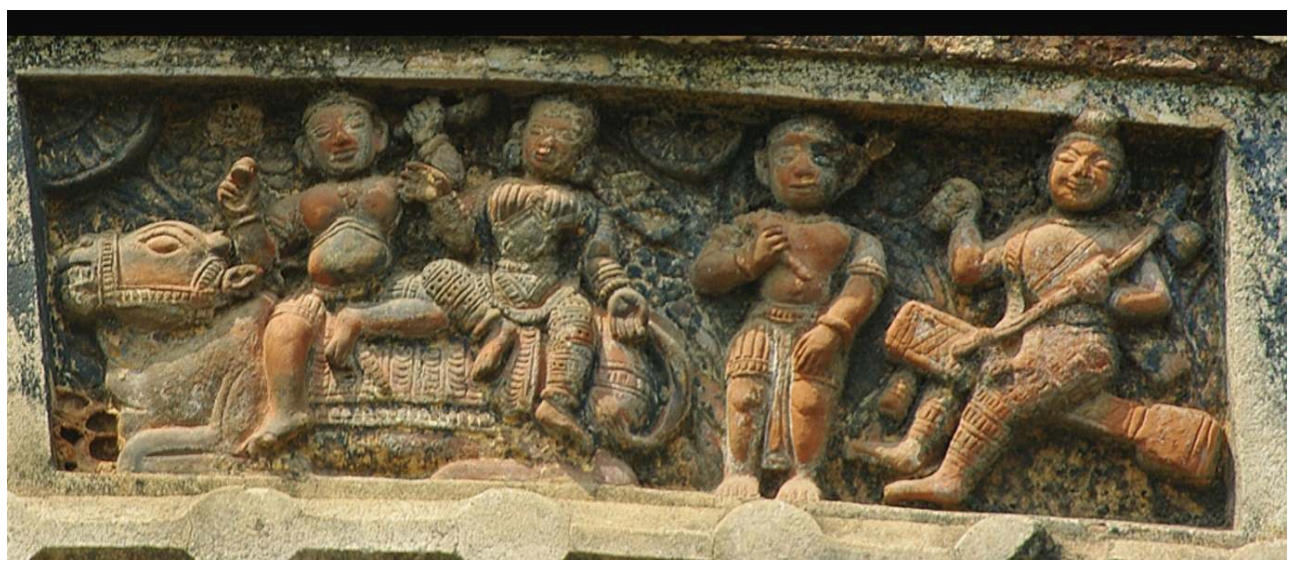

Radha-Damodar Navaratna Mandir, pannello su primo piano della facciata orientale (c. xIx secolo), Dattapara, Jaypur, Bishnupur Distt. (WB). Sanathana e Hazarika 2020, 9.

Fig. 7. Nārada a cavallo del ḍ̂ẽ̃ī con vīṇā (mano sinistra) e kartāl (mano destra).

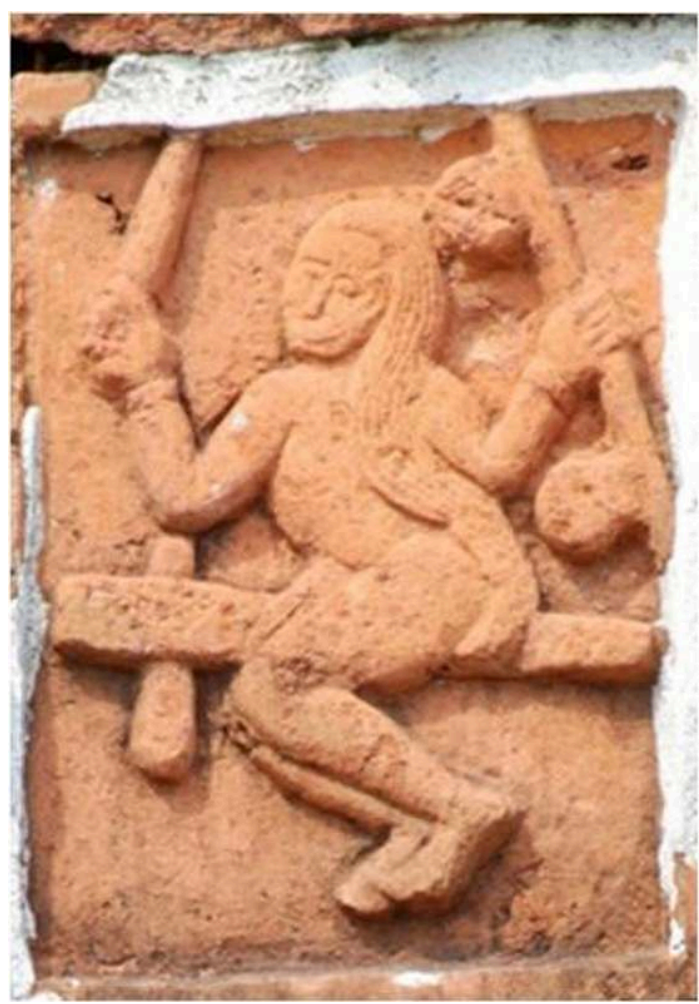

Targa in terracotta, portale meridionale, Ghanashyam's House (xvII secolo), Joysagar, Shivasagar Distt. (Assam). Sanathana e Hazarika 2020, 9. 
Fig. 8. Nārada su ḍhẽkī con gandharva al seguito.

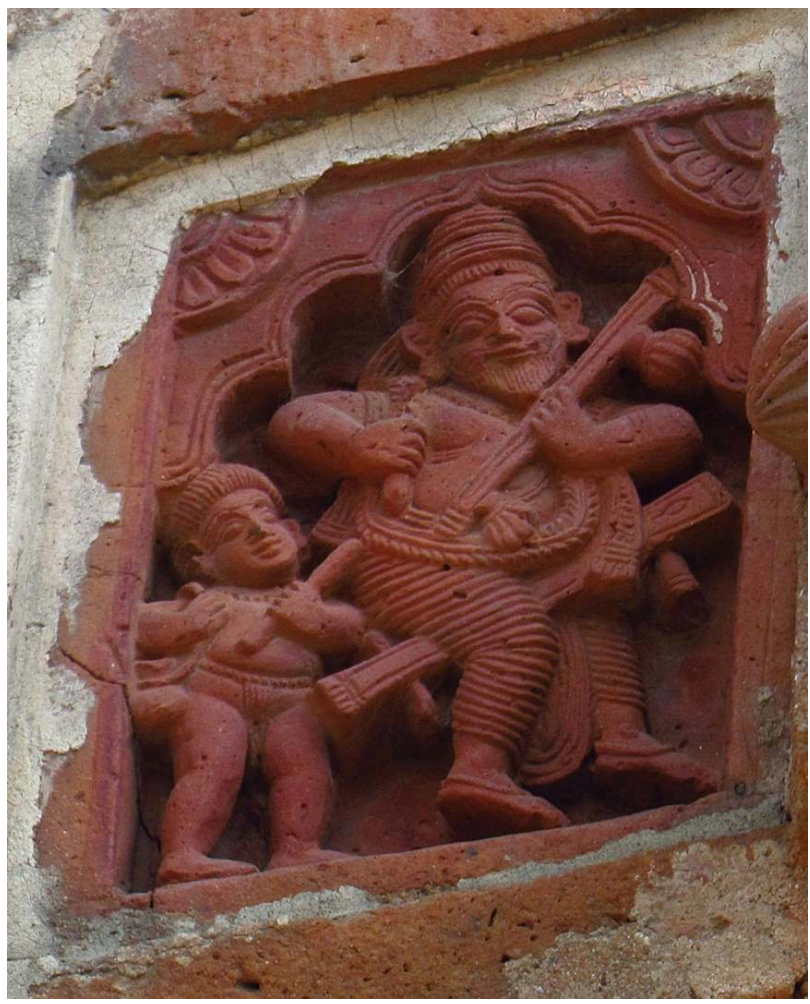

Shiva Mandir n. 3 (1768), Uchkaran, Birbhum Distt. (WB). Sanathana e Hazarika 2020, 9

A differenza del Gājan, i riti associati al dhếkī, probabilmente in quanto legati alla cultura votiva delle donne (mejeder brata), non presentano alcun collegamento con la tradizione testuale di Dharmarāj o di Śiva. Sebbene occasionalmente inseriti nel contesto celebrativo del Gājan, tale ritualismo rappresenta una tradizione le cui radici affondano nei culti della natura. Allo stesso modo, il ḍhêkimañgal che troviamo in ŚP e i d̦hêki gān cantati oggi dalle donne bengalesi rappresentano un momento lirico e sociale limitato dal contesto di genere e occupazionale che solo parzialmente cede spazio a divinità indigene o purāniche.

\section{Considerazioni storiche}

31 La tesi secondo cui il cāṣāpālā in ŚP sarebbe una tarda interpolazione legata al processo di sanscritizzazione di tradizioni religiose regionali (āñcalik), nello specifico di Rạaṛh, presenta a nostro avviso importanti incongruenze di tipo storico e ambientale alla luce delle quali si potrebbe riconsiderare non solo la datazione di ŚP ma anche il contesto geografico e culturale di origine. Muoveremo da due considerazioni congiunte: (i) la mitografia di Rāmāi Paṇdit non trova riscontro nelle tradizioni letterarie del Bengala occidentale; e (ii) le colture e i metodi di coltivazione descritti nel mito della nascita del riso non corrispondono alla struttura idrogeologica di Rāarh.

Nei testi a lui attribuiti, Rāmāi tace circa l'epica di Lāusen, l'eroe nazionale di Rāṛh, le cui gesta sono celebrate nella ricca tradizione dei Dharmamangal' $k a ̄ b y a^{49}$. Questo filone epico muove dalla sconfitta in battaglia di Karṇasen, un vassallo del re di Gaur (Gauṛeśvar), per mano di Ichāi Ghoș, un signorotto appartenente alla casta pastorale 
dei goyāl. Una volta spodestato, Karṇasen, i cui figli erano stati uccisi in guerra, perde anche la moglie. Sposa quindi la giovane principessa Rañjābatī, figlia del re di Gaur, con la speranza di ottenere una discendenza e quindi garantire un futuro alla sua dinastia. Tuttavia, per via dell'età avanzata, i tentativi di concepire un figlio falliscono. Rañjābatī viene allora consigliata dalla sua balia ḍom di pregare Dharmarāj, una divinità legata a questa casta "impura", e di rivolgersi a Rāmāi Paṇdit, il "sacerdote" di Dharma Țhākur ${ }^{50}$. Questi accompagna la regina sulle rive del fiume Dwarakeshwar, presso il Chāmpai Ghāt, per una serie di austerità devozionali. Anche in questo caso, la regina non riesce a rimanere incinta e pertanto decide di offrire la propria vita a Dharmarāj». Nell'episodio noto come śăle bhar, ${ }^{51}$ Rañjābatī muore ma, grazie alla sua profonda devozione, è riportata in vita e resa madre da Dharmarāj. Nascerà Lāusen, l'eroe che dopo una serie di avventure a metà tra il Bildungsroman e il poema epico - riconquisterà il trono del padre: inizialmente sconfitto con il suo esercito di guerrieri dom, Lausen invoca Dharmarāj e, promettendogli l'offerta di un nabakhaṇda, un rito secondo cui il corpo della vittima sacrificale, in questo caso egli stesso, è tagliato in «nove parti», gli chiede di far sorgere il sole ad ovest (paścim udaỳ) e di resuscitare i suoi guerrieri. Dharmarāj accetta il sacrificio di Lāusen, lo riporta in vita insieme al suo esercito e ne consente la vittoria finale.

Questo grande affresco epico, peraltro centrale nel ritualismo marziale del Gājan, è del tutto assente in ŚP, dove Rāmāi svolge il ruolo di officiante alla corte di un tale «Haricandrarāja» che, insieme alla consorte Madanā, è lo sponsor di una grandiosa Dharmapūja ${ }^{52}$. Proponiamo di identificare tale figura con Hariścandra di Sābhār (Dhaka), figlio di Ranadhïra Sena ${ }^{53}$. Tale tesi pare trovare riscontro in un'iscrizione rinvenuta tra le rovine di un monastero (mathha) e di uno stūpa presso Majidpur (distretto di Dhaka), un sito noto come Hariścandra Rājār Prasād Ḍhibi e datata 791 Śaka (=869 d.C. $)^{54}$, in cui è menzionato l'arrivo di Dhīmanta Sena «nella regione di Bhābalīna (=Bhāoyjāl; Bhawal) ricca di colline e foreste» (āyātismādrivanalasite bhābalīne pradeśe; v. 1):

2. vamisāvatībrahmasutapravișțam

dakṣeṇa gāngam sa ca bhābalīnam /

dhìmantasenah saha sainyayodhai

rātrāmatisnā prabalāt kirātāt //

3. dhīmantaputro ranadhīrasenah

samingāmagetā iva kārttikeyaḥ /

himālayavyāptadeśān vijitya

sambārapurjyāmavasat pravīrah //

hariścandro mahārājah raṇadhīrasya putrakaḥ /

4. dharmeśa iva dharmātmā dhanāṭyah kuberādhikah //

5. [...]

6. yamalātrāsinī tīre bauddhāskamațhamandire /

vijane ca sa rājarșị̣ dharmārtham snābatișțhate //

2. Dhīmanta Sena, [discese alla testa di] soldati e guerrieri dal [territorio dei] Kirāta e conquistò quel tratto di Bhābalīna che giace tra [i fiumi] Brahmaputra e Vamísāvatīi, a sud dei quali [scorre] il Gange.

3. Il valoroso eroe Ranadhīra Sena, figlio di Dhīmanta, vittorioso in battaglia come il dio Kārttikeya, dopo aver conquistato la regione fino all'Himalaya, stabilì la sua residenza nella città di Sāmbara [Sābhār].

4. Re Hariścandra, figlio di Raṇadhīra, si dimostrò incline alla pietà come Dharmeśa

[= Buddha] e Kubera, il Signore delle ricchezze [...]».

5. [...] 
6. [...] Per acquisire merito, questo divino patrono [Hariścandra] [...] soggiornava presso monasteri isolati e templi decorati con immagini del Buddha sulle rive degli affluenti del fiume Jamuna (yamalātrāsinī). Tuttavia, fonti letterarie ed epigrafiche confermerebbero la presenza di un re Hari(ś)candra a Sābhār nel IX secolo. Secondo i Maynāmatīr gān e Gopicandrer Gān («I canti di Maynāmatī» e «I canti di Gopicānd») $)^{55}$, una serie di composizione śivaite legate ai nāth del Bengala orientale, questi sarebbe il padre delle principesse Adunā e Padunā che, grazie all'intervento della regina Maināmatī, andarono in moglie a Govindacandra (=Gopīcand), ultimo sovrano della dinastia Candra, che l'iscrizione di Tirumalai (datata 1025) descrive come il re della regione detta Vangāladeśa, rovinosamente sconfitto insieme a Dharmapāla I, Raṇasūra di Dakșina Rāạha e Mahīpāla di Uttara Ṛārha da Rajendracola, della dinastia Tamil dei Cola ${ }^{56}$.

Si noti come la più tarda leggenda che troviamo nei Dharmamañgal'kābya sia ricalcata su quella nāth: un sovrano sconfitto e impossibilitato a procreare (Manikcānd; Karnasen) costringe una regina (Maināmatīi Rañjabatīi) ad un suicidio rituale (sātīi śâle bhar) per ottenere un figlio che viene concesso grazie all'intervento di una figura semidivina (Gorakhnāth; Rāmāi Panditit). Nasce quindi l'erede (Gopīcand; Lāusen) che permetterà la diffusione del culto di Śiva/Dharmarāj rispettivamente nel Bengala orientale (Rangpur, Dhaka) e occidentale (Rāṛh) grazie a pratiche di tipo ascetico.

Passiamo ora al secondo punto. Oltre a permetterci di datare «Hari(ś)candrarāja», l'iscrizione di Majidpur presenta una serie di preziosi indizi ambientali che ci permettono di definire con una certa precisione l'area governata da Hariścandra. Si tratterebbe di una zona compresa tra le odierne divisioni di Dhaka e Mymensingh e delimitata a est, dal basso Brahmaputra (la rete di canali della Buṛi Brahmaputra); ad ovest, dalla Vamísāvatī (= Bangsśî, Bangshi) (vamiśāvatībrahmasutapravistami); a nord, dalle pendici himalayane (= il territorio dei Kirāta); e a sud dalla Buṛī Gañgā (dakșena gāngamin) (Fig. 9). In breve, abbiamo qui a che fare con la frontiera orientale dell'area di influenza brahmanica, un territorio che era già inteso di confine nell'epoca tardo-vedica e che tale rimarrà fino al periodo moderno. La frontiera nord-orientale, non è però da intendersi come il limite che denota un aldilà barbarico. Si tratta, come vedremo, di un orizzonte geografico e culturale per sua natura mobile, in quanto dipendente dalle acque $^{57}$, in cui convergono lingue e tradizioni diverse ${ }^{58}$. 
Fig. 9. Fiumi del Bangladesh

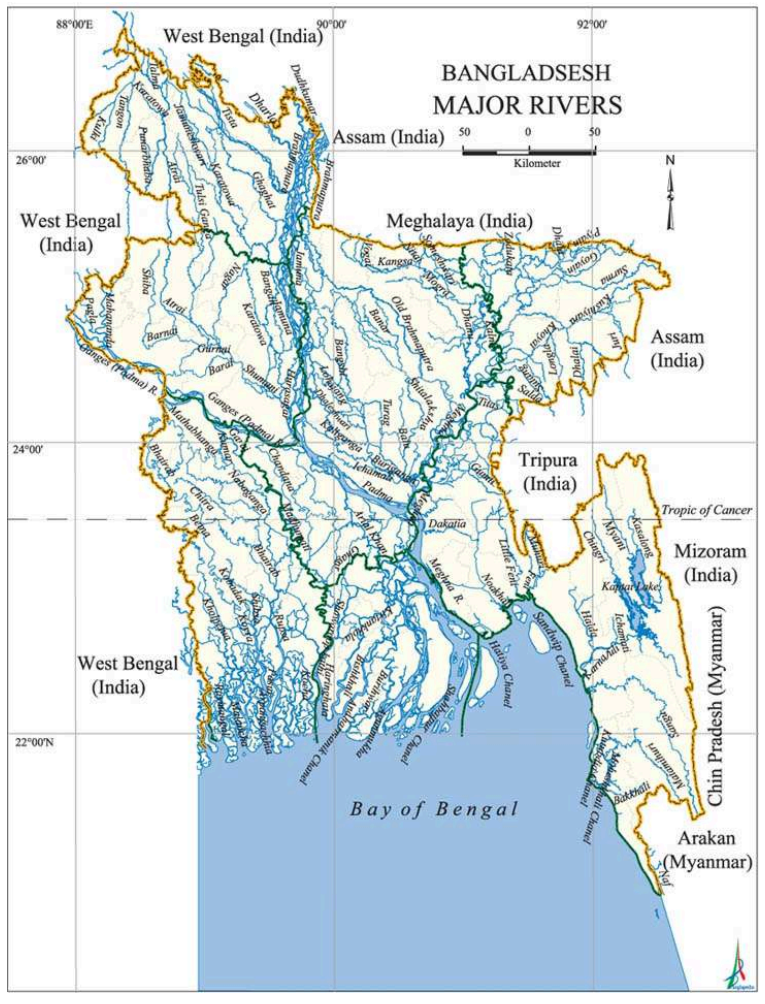

Wikimedia

37 Oltre alla presenza di Hariścandra nei pressi di Dhaka e all'esistenza di una tradizione rituale śivaita speculare al Gājan di Dharmarāj, la Nïl'pūja dedicata a Buṛa Śib («Vecchio Śiva» $)^{59}$, altre considerazioni ci permetterebbero di identificare quest'area con quella descritta nel mito della nascita del riso in ŚP. Da un lato, la pratica del debbio (jhum) di cui ci parla Rāmāi è estranea a Rạậh mentre è stata a lungo praticata nel nord e nordest del Bangladesh, il cui panorama era ancora selvaggio e coperto di foreste impenetrabili ${ }^{60}$. Dall'altro, la coltivazione del riso in sommersa va analizzata tenendo in considerazione una serie di rilievi ambientali.

In primo luogo, è importante considerare che il corso e la portata del Gange (Ādigangāa) e i suoi effluenti, Jamuna (= basso Brahmaputra) e Saraswati, un canale distributivo del Bhagirathi oggi scomparso, subirono nel periodo medievale importanti cambiamenti che portarono alla formazione del complesso Padma-Meghna ${ }^{61}$ in Bangladesh e causarono ad ovest siccità, epidemie, carestie. Già nell'eulogia di Bhațta Bhavadeva «Bālavalabhïbhujanga», un'iscrizione rinvenuta presso il tempio di Ananta Vāsudeva a Bhubaneshwar (Odia) e datata XII secolo, troviamo un riferimento a Rāṛh come una zona "priva di acqua» (rāạhāyāmajalāsu jāngalapatha, v. 18) ${ }^{62}$, che pertanto non può essere la sede delle peregrinazioni di Haricandrarājā e Rañjābatī, come indicato nei Dharmamangal. Tale informazione è confermata da recenti rilievi storici e geologici che individuano l'intera area come paesaggio lateritico inadatto all'agricoltura e di difficile sfruttamento per tutte quelle attività che necessitano di sostanziale apporto d'acqua (Fig. 10) ${ }^{63}$. 
Fig.10. Mappa fisica del bacino gangetico nel Bengala occidentale. Si vedano in particolare le caratteristiche di Rāạh (aree II e III).

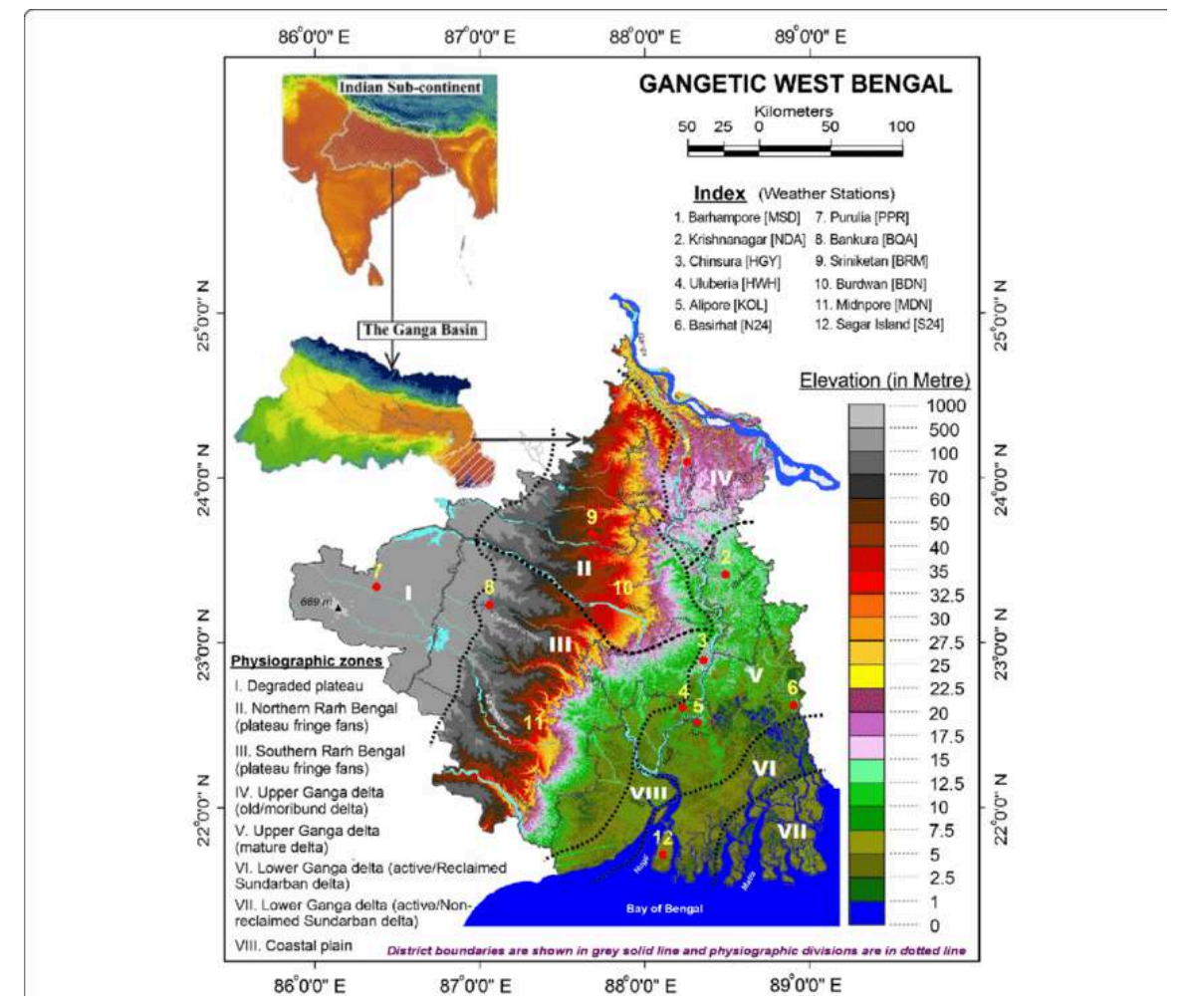

GHOSH 2018, 3

La drammatica mancanza di sufficienti risorse idriche portò al collasso numerosi porti fluviali di grande importanza tra cui Tāmralipta (Tamluk) e Saptagrām. Si veda al proposito la testimonianza del mercante veneziano Cesare Federici (c. 1530 - 1603): «[...] prima che fi arrivi a Satagan [=Saptagrām/Sātgão], fi troua vu luoco che fi chiama Bettor (=Betar), e da lì in fu non vanno le naui grofle, perch'il fiume ha poca acqua [... $]^{64 » . ~ I l ~ f i u m e ~ d i ~ c u i ~ F e d e r i c i ~ p a r l a ~ e ̀ ~ i l ~ B a g h i r a t h i-H o o g h l y ~(G a n g e, ~ o ~ K a ̄ t ̣ i g a n ̃ g a ̄), ~ l a ~ c u i ~}$ portata era già significativamente ridotta per via del progressivo diseccarsi della Saraswati e della Jamuna. Infatti, quando nel 1565 Munim Khān, primo ministro dell'impero Moghul e governatore del Bengala, spostò la capitale da Tāṇ̣ā a Gaur, nel breve giro di circa dieci anni fu costretto a ritirarsi in quanto lo spostamento del bacino idrico aveva causato un ambiente acquitrinoso e malsano al punto da favorire lo scoppio della peste nel $1575^{65}$. Le mappe del cartografo portoghese João Baptista Lavanha (1550-1624), del geografo fiammingo Petrus Bertius (1525-1629) e di Mattheus van den Broucke, direttore della Compagnia olandese delle Indie orientali di stanza in Bengala (1658-1663), mostrano chiaramente il progressivo squilibrio tra la portata dei sistemi idrici del Bengala orientale rispetto al complesso del Bengala occidentale (Figg. 11-13) ${ }^{66}$, fatto peraltro confermato dallo spostamento a Dhaka della sede del Bānglā Subāh, la partizione amministrativa orientale dell'Impero Moghul. 
Fig.1 1. Il golfo del Bengala, in particolare l'area tra Betore (Betar) e Dhaka (Dhākā), e il corso del Rio Caor (=Buṝ Brahmaputra) Enseada de Bengalla, di João Baptista Lavanha.

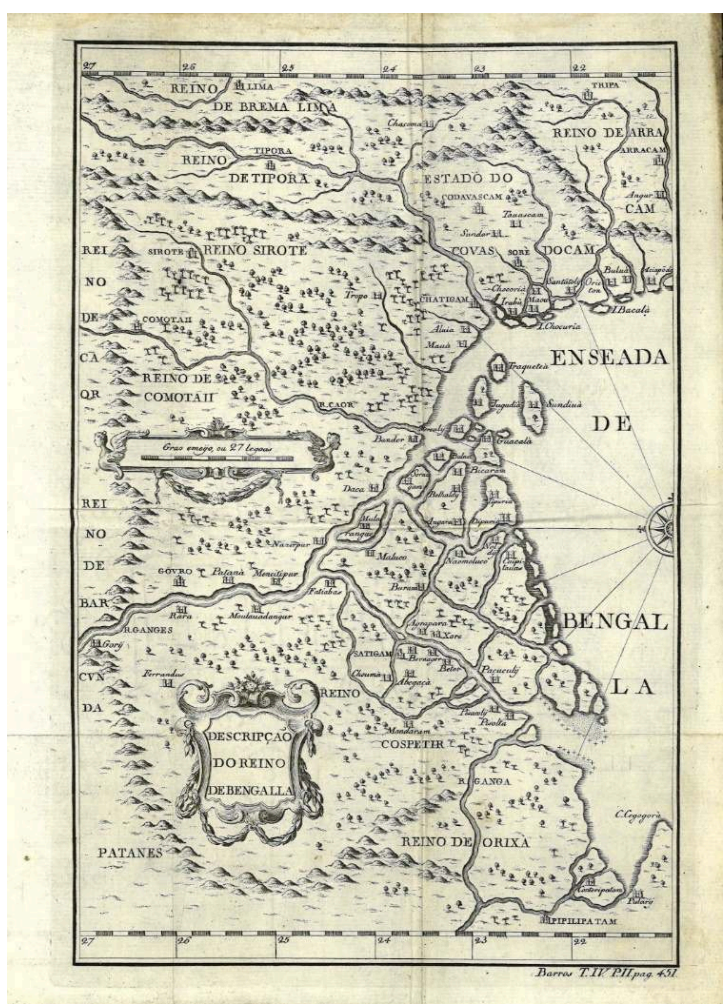

de Barros 1575

Fig. 12. Tabularum Geographicarum Contractarum, Petrus Bertius, Amsterdam, 1618.

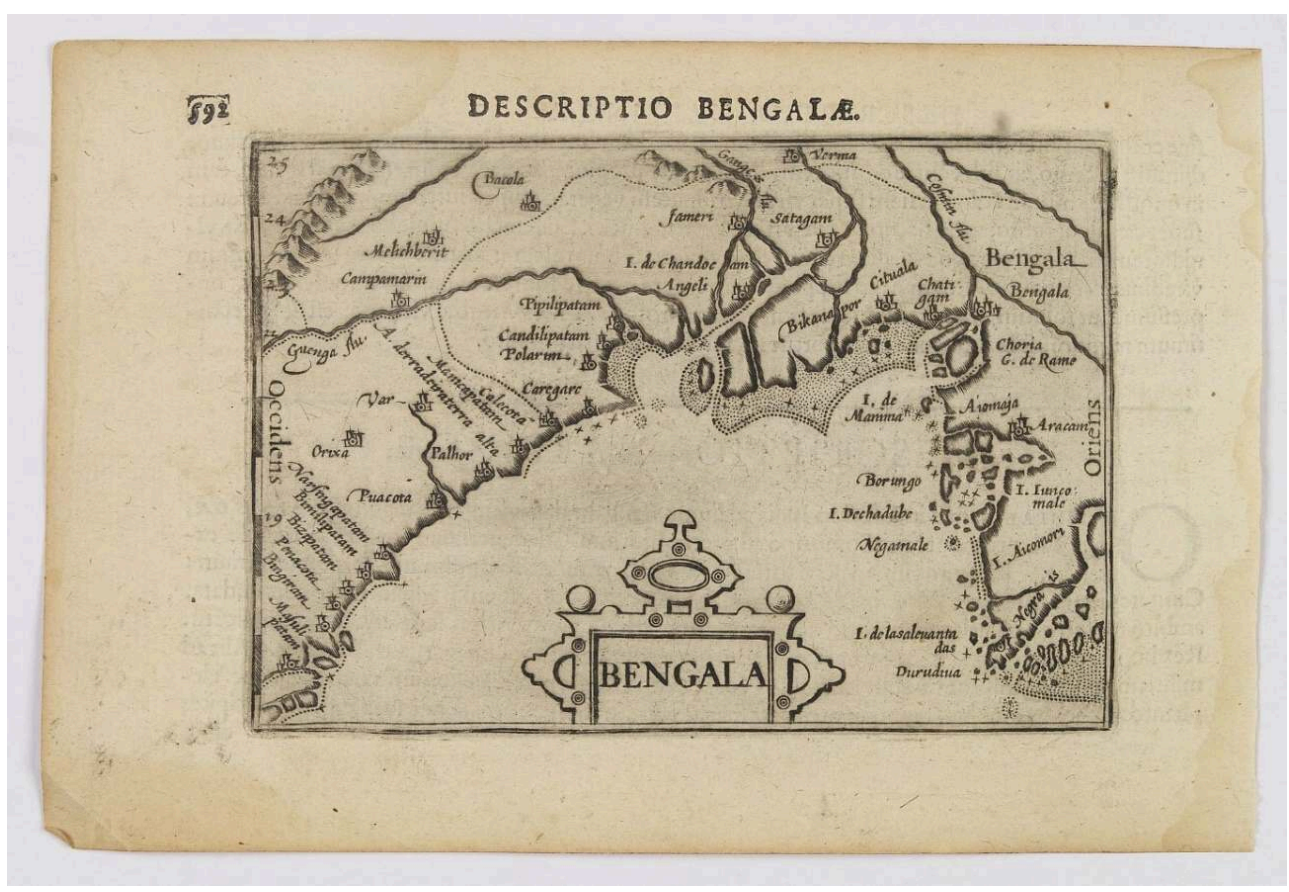

Bertius 1616 
Fig.13. Particolare, Mappa del Bengala, 1660, di Mattheus van den Broucke.

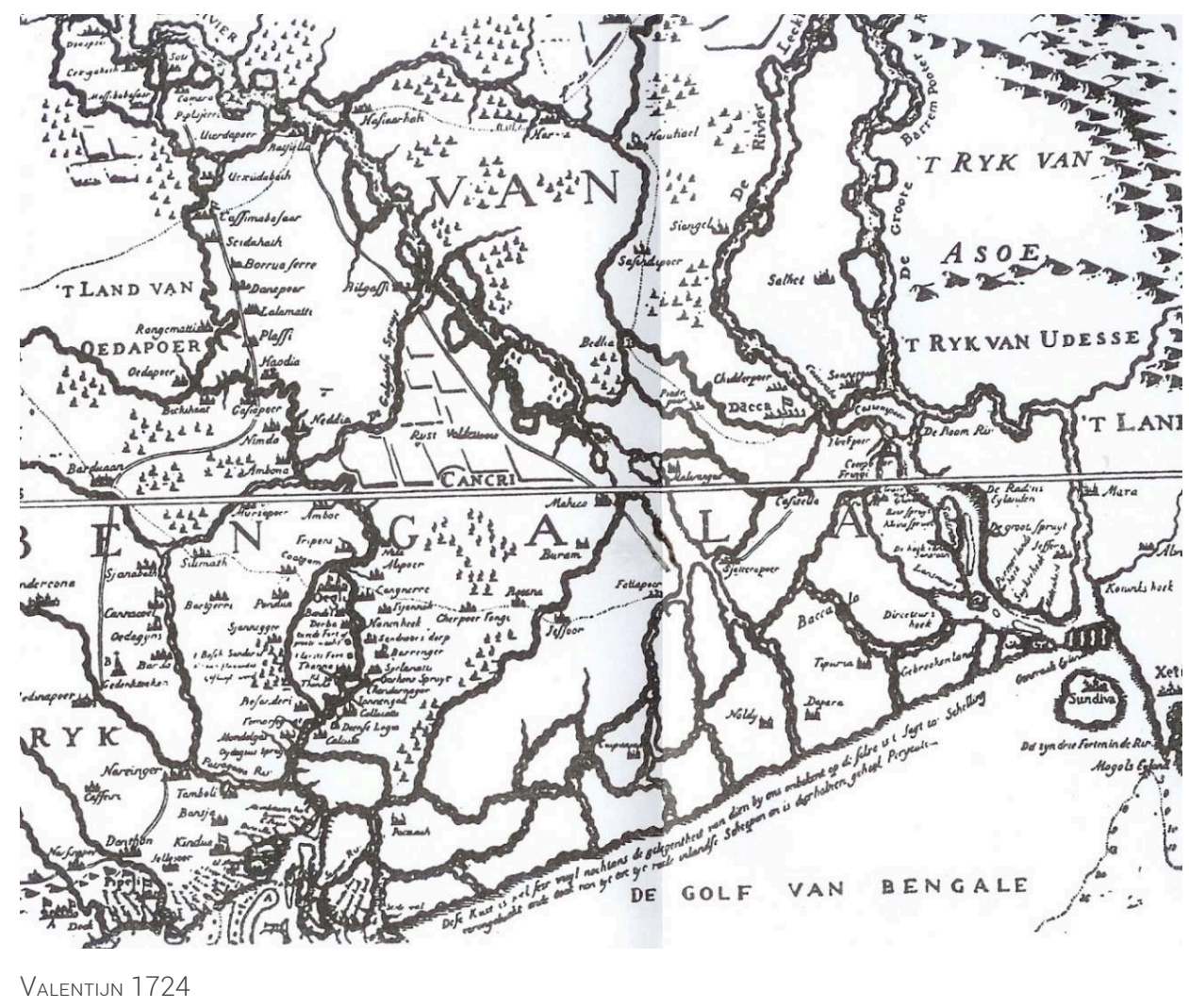

In considerazione di ciò, il riferimento al jalidhān («riso galleggiante») in ŚP si rivela incongruente. Le varietà di deepwater rice, proprio come indicato nel testo, necessitano che il terreno sia preparato a partire da gennaii ${ }^{67}$ per poi essere seminate tra giugno $\mathrm{e}$ luglio ${ }^{68}$ in modo da crescere fino a metà agosto e sopportare le successive inondazioni ${ }^{69}$. Tali varietà trovano il terreno ideale nei terreni alluvionali del Bangladesh mentre è stato osservato che le aree progressivamente collinose del Bengala occidentale a partire dal distretto di Barddhaman procedendo verso sud, una zona parzialmente congruente con la regione di Rạaṛh, rende impossibile la coltivazione del riso galleggiante ${ }^{70}$.

41 Il Bengala orientale, spesso identificato come terra di frontiera vedica e brahmanica prima e islamica dopo, con riferimento principalmente alla cosmopoli turco-mongola dei Moghul, dovrebbe essere in realtà considerato come l'incontro di più frontiere, ciascuna diversamente denotata dalla propria relazione con la mobilità, spesso imprevedibile, dei sistemi idrici e delle maree. Le rotte commerciali, così come le migrazioni di popoli, hanno così favorito più che altrove il continuo fluire di tecniche $\mathrm{e}$ sistemi di conoscenza la cui unicità e interpretazione trova riscontro nelle tradizioni letterarie bengalesi quali kathā, mangal, kābya, sangit, yātrāa, ecc. e, più in generale, nelle arti. Il mito della nascita del riso riflette esattamente il paradigma della tradizione di frontiera che, proprio in quanto tale, assurge a narrativa cosmopolita.

\section{Conclusione}

Nelle pagine precedenti abbiamo proposto un'analisi alternativa dei materiali relativi al mito della nascita del riso narrata da Rāmāi Paṇdit. Questo particolare approccio 
muove da un'analisi delle varianti manoscritte dello śūnyapurān ma anche di documenti letterari quali i Dharmamangal' kābya, la letteratura Śibāyan e fonti epigrafiche. Infine, riscontri ambientali legati al panorama idrogeologico del Bengala medievale si sono rivelati di grande utilità per meglio contestualizzare i riferimenti ambientali interni. Sulla base di questi dati è stato possibile trarre importanti conclusioni circa la geografia religiosa di Rāarh e del Bengala orientale. In entrambi i territori si osservano festival annuali legati al ciclo della terra con caratteristiche simili: da un lato, il Gäjan di Dharmarāj, una divinità la cui prassi rituale è dettagliata da Rāmāi Paṇdit e il cui potere è cantato nell'epica di Lāusen dei più tardi Dharmamañgal'kābya; dall'altro la Nîl'Pūjā o Siber gājan, un festival legato al folklore contadino e alle leggende nāth sviluppatesi in seno alle comunità agricole orientali. Si noti anche che l'epica popolare di Lāusen presenta una struttura narrativa del tutto sovrapponibile alle agiografie dei nāth del bengala orientale, in particolare le vicende di Maināmatī e Gopīcānd, padre di Hari(ś)cāndra. Quest'ultimo potrebbe inoltre essere il patrono di Rāmāi Paṇdit, fatto che ci permetterebbe di collocarlo nella seconda metà del Ix secolo nei pressi di Dhaka. A ciò va aggiunto che la descrizione delle colture (deepwater rice), le tecniche di coltivazione del riso (asciutta preceduta da debbio e sommersa) e la struttura idrogeologica di Rạaṛh non corrispondono con i dati che estrapoliamo da ŚP. È pertanto possibile che il culto dello Śiva contadino si sia inizialmente sviluppato nel Bengala orientale, nei distretti a nord e ad est di Dhaka, dove potrebbe aver operato Rāmāi stesso intorno al IX secolo. La caduta della dinastia Pāla con conseguente declino del buddhismo, l'arrivo dei Sena ${ }^{71}$ prima e dell'islam intorno al xII secolo sono ulteriori elementi da considerare riguardo la migrazione di alcuni elementi dello śivaismo orientale nel Bengala occidentale. Se questa ipotesi fosse confermata, il culto di Dharmarāj dovrebbe essere riletto come una forma di śivaismo vernacolare adattata al contesto culturale di Rạaṛ e, solo in tempi più recenti, riconvertita in quello śivaismo sanscritico che trova la sua massima forma di espressione nella letteratura purāṇica. Analizzate in questo modo, la cultura e la letteratura del Gäjan vanno ripensate come riti di fertilità originati all'interno di potentati locali e influenzati dal folklore nāth e originariamente sviluppatesi nel contesto di un'epopea di frontiera legata alla colonizzazione e bonifica delle foreste e delle piane alluvionali del Bengala orientale.

BIBLIOGRAFIA

AN (Akbarnāma): The Akbar Nāma of Abu'l Fazl (History of the Reign of Akbar Including an Account of His Predecessors), Translated from the Persian by Henry Beveridge (Reprint, 1939), 3 voll., Bibliotheca Indica 138, Calcutta 2000.

AVŚ (Atharvaveda, Śaunakasaṃhitā): C. Orlandi (a c. di), Gli Inni dell'Atharvaveda (Śaunaka), Pisa 1991.

BASU 1316 BS: N.N. Basu, «Śūnyapurāṇ sambondhe mantobya», Sāhitya Parișat Patrikā, 16/4 (1316 BS), 221-224. 
De Barros 1575: J. de Barros, Década Quarta da Ásia. Dos feitos que os Portugueles fezerão no descobrimento, e conquista dos mares, e terras do Oriente, Lisboa, 1575.

BHAKTIBINODE 1313 BS: B.K. Bhaktibinode, «Rāmāi Paṇdit o Maynāpurer Yātrāsiddhi». Sāhitya Parișat Patrikā, 13/2 (1313 Bs), 81-96.

BERTIUS 1616: P. Bertius, Tabvlarvm geographicarvm contractarvm libri septem. In quibus tabuloe omnes gradibus distincte, descriptiones accuratoe, ccetera supra priores editiones politiora, auctioraq[ue], ad christianissimum Gallice \& Navarrae regem Lvdovicvm XIII. Amsterodami 1616.

BHATṬ̂̄CĀRYA 1959: Ā. Bhațtācārya, Bāninglā Mañgal'kābyer Itihās (pañcadaś-șoḍaś śatabdī, Triotiỷa saṃskaraṇ, Kalikātā 1959.

BHAṬṬĀCĀRYA 1961: Ā. BHAṬṬĀCĀRYA (samp), Gopīcandrer Gān, Tṛtiỷa saṃskaraṇ, Kalikātā 1961. BHATṬ̂̄CĀRYA 2000: Ā. Bhațțācārya, Bānglā Mañgal'kābyer Itihās, Dbādaś saṃskarạ, Kalikātā 2000. BHAṬṬĀCĀRYA 1951: Ā. Bhațțācārya, The Early Saiva Bengali Poetry (Reprint 1944 edition), Calcutta 1951.

BHATtASAli 1920: N. K. Bhattasali, «The Math inscription of Mahendra, Son of Harish Chandra of Sabhar», Dacca Review, 10/6-7 (1920), 111-115.

CATLING et al. 1983: H.D. Catling, P.R. Hobbs, Z. Islam, B. Alam, «Agronomic practices and yield assessments of deepwater rice in Bangladesh», Field Crops Research, 6 (1983), 109-132.

ChAKRABARTY e MANDAl 2019: P. Chakrabarty, R. Mandal, «Geoarchaeosites for geoturism: a spatial analysis for Rarh Bengal in India», GeoJournal of Tourism and Geosites, 25(2), 543-554.

Chattopadhyaya 1997: D.P. Chattopadhyaya (ed.), Tāranātha's History of Buddhism in India (Reprint), Delhi 1997.

CLARK 1955: T.W. Clark, «Evolution of Hinduism in Medieval Bengali Literature: Śiva, Candīi, Manasā», Bulletin of the School of Oriental and African Studies, 17/3 (1955), 503-518.

CLARKE 1888: C.B. Clarke, «The Cultivation of Rice in Bengal», Bulletin of Miscellaneous Information (Royal Gardens, Kew), 1888/24 (1888), 284.

CM (Caṇịmañgal): D.C. Sen, H. Bandyopādhyāẏ, N. Basu (sam.), Kabikañkaṇ'Caṇdī, Kalikātā 1924.

CRRI 2012: Indian Agricultural Research Institute, New Delhi, Krșikoś, An Institutional Repository of Indian National Agricultural Research System, Central Rice Research Institute, New Delhi: https:// 14.139.56.90/ (ultimo accesso 28 marzo 2021).

CURLEY 2008: D.L. Curley, «Battle and Self-Sacrifice in a Bengali Warrior's Epic: Lāusen's Quest to be a Raja in Dharma Mańgal», in R.W. Nicholas (ed.), Rites of spring: Gājan in Village Bengal, Delhi 2008, 142-219.

D'HUBERT 2018: T. D'Hubert, In the Shade of the Golden Palace: Ālāol and Middle Bengali Poetics in Arakan, South Asia Research, New York, 2018.

DAS GUPTA 1929: T.C. Das Gupta, «Aspects of Bengali Society (Agriculture)», Journal of the Department of Letters 18 (1929): 181-216.

DASGUPTA 1955: S.B. Dasgupta, Obscure Religious Cults (3. ed., reprint 1946), Delhi 1955.

DHM ${ }^{\mathrm{GH}}$ : Y.C. Basu (sam.), Śrīdharmamangal. Śrī Ghan'rām Cakrabarttī Kabiratna Pranìt, Kalikātā 1290 BS. 
DHP (Dharmapurāṇ): B.K. Cațtopādhyāẏ (sam.), Śrīdharmapurān. Maỷūr Bhatța-biracit, Kalikātā 1337 BS.

DHPB (Dharmapūjābidhān): N.G. Bandyopādhyāẏ (sam.), Dharmapūjābidhān. Śrīrāmāi Paṇdit-biracit, Kalikātā 1323 BS.

DNM (Deśināmamālā): R. Pischel (ed.), The Deśināmamālā of Hemacandra, Second edition with introduction, critical notes, and glossary by P.V. Ramanujaswami, Bombay 1938.

DRD 2013: DACNET Project, Dept. of Agriculture and Cooperation, Directorate of Rice Development, Patna: http://www.drdpat.bih.nic.in/ (ultimo accesso 28 marzo 2021).

EATON 2000: R.M. Eaton, The Rise of Islam and the Bengal frontier, 1204-1760, $3^{\text {rd }}$ Impression, New Delhi.

EI VI: F. Kielhorn, «Two Bhuvanesvar inscriptions », Epigraphia Indica, VI (1900/1901), 198-207.

EI IX: E. Hultzsch, «Tirumalai rock inscription of Rajendra-Chola I», Epigraphia Indica, IX (1907/1908), 229-233.

EI XV: R. Basak, «Tipperah copper-plate grant of Lokanatha: the $44^{\text {th }}$ year», Epigraphia Indica, xv/1 (1919/1920), 301-315.

EI XXI: R. Bhandarkar, «Mauryan Brahmi Inscription of Mahasthan», Epigraphia Indica, XXI (1931/1932), 83-91.

FEDERICI 1587: C. Federici, Viaggio di M. Cesare de i Federici, nell'India orientale, et oltra l'India: Nelquale fi contengono cofe diletteuoli de i riti, \& de i co/tumi di quei paefi, et infieme fi defcriueno le fpetiarie, droghe, gioie, \& perle, che d'effi fi cauano. Con alcuni auertimenti vtiliffimi a quelli, che tal viaggio volefsero fare, Venezia 1587.

FERRARI 2010: F.M. Ferrari, Guilty males and proud females: Negotiating genders in a Bengali festival, Calcutta 2010.

FERRARI 2015: F.M. Ferrari, « "Illness Is Nothing But Injustice”: The Revolutionary Element in Bengali Folk Healing», Journal of American Folklore 128/507 (2015), 46-64.

FERRARI 2016: F.M. Ferrari, «Agriculture, Floriculture and Botanical Knowledge in a Middle Bengali Text», in F. M. Ferrari, T.W.P. Dähnhardt (eds.), Roots of Wisdom, Branches of Devotion: Plant Life in South Asian Traditions, London, 241-269.

GopAl e SRIVASTAVA 2008: L. Gopal e V.C. Srivastava (eds.), History of Agriculture in India, up to c. 1200 A.D., History of Science, Philosophy, and Culture In Indian Civilization. Volume V. Agriculture in India, pt. 1., New Delhi, 2008.

GHosн 2015: K. G. Ghosh, «Analysis of Rainfall Trends and Its Spatial Patterns During the Last Century over the Gangetic West Bengal, Eastern India», Journal of Geovisualization and Spatial Analysis, 2/15 (2018), 1-18.

GRIERSON 1878: G.A. Grierson, The Song of Mánik Chandra (Re-printed from the Journal of the Asiatic Societyof Bengal, Part I, no. 3, 1878), Calcutta 1878.

GRIERSON 1926: G.A. Grierson, Bihar peasant life: Being a discursive catalogue of the surroundings of the people of that province (Second revised edition), Patna 1926.

KAR et al. 2012: G. Kar, N. Sahoo, A. Kumar, «Deep-water rice production as influenced by time and depth of flooding on the east coast of India», Archives of Agronomy and Soil Science, 58/6 (2012), 573-592. 
KKS (Kāśyapīyakrșisūkti): G. Wojtilla (ed.), Kāśyapiyakrșisūkti. A Sanskrit Work on Agriculture, Wiesbaden 2010.

KP (Krșiparāśara): D.P. Śāstrī (ed.), Parāśaramuniviracitạ̣ Krșiparāsáarah. With Hindī translation, Vārāṇasī 2003.

LEWIN 1869: T.H. Lewin, The Hill Tracts of Chittagong and the Dwellers Therein; with Comparative Vocabularies of the Hill Dialects, Calcutta 1869.

LEWIN 1912: T.H. Lewin, A Fly on the Wheel, or How I Helped to Govern India, London 1912.

LUDDEN 1999: D.E. Ludden, An Agrarian History of South Asia, The new Cambridge History of India, IV , 4, Cambridge, 1999.

MANRING 2018: R.J. Manring, "Child Sacrifice in Rūparāma's Dharmamanigala», The Journal of Hindu Studies, 11/3 (2018), 187-206.

MANU: F. Squarcini; D. Cuneo (a c. di). Il trattato di Manu sulla norma (Mānavadharmaśāstra), Torino 2010.

MITRA 1972: A. Mitra, Rāạhher saṃskṛti o Dharma Ṭhākur (Bīrbhūmite prāpta tathyāloke), Kalikātā 1972.

MUKHOPĀDHYĀẎ 1280 BS: K. Mukhopādhyāẏ, Matsyadharā Nāțak, Kalikātā1280 BS.

NoLAN 1859: E.H. Nolan, The Illustrated History of the British Empire in India and the East from the Earliest Time to the Suppression of the Sepoy Mutiny in 1859, illustrated with steel engravings and maps, vol. I, London, 1859.

PASTENA 2017: C. Pastena, Il libro asiatico, Palermo 2017.

RĀỶ 1326 BS: J.C. Rāỷ, «Śūnyapurāṇ», Sāhitya Parișat Patrikā, 16/4 (1326 BS), 203-220.

RĀỶ 1338 BS: J.C. Rāỳ, «Śūnyapurāṇ», Sāhitya Pariṣat Patrikā, 38/2 (1338BS), 65-91.

RAỶ 1420 BS: N. Raẏ, Bāngālīr Itihās. Ādi parba, așțam saṃskaraṇ, Kalikātā 1420 BS.

RRI 1980: The Reporter on Economic Products to the Government of India (ed.), Races of Rice in India. Reprint of 1911 edition. Original title: The Agricultural Ledger, New Delhi 1980.

ŚAHĪDULLĀH 1360 BS: M. Śahīdullāh, «Maȳūr Bhațța», Sāhitya Parișat Patrikā, 60/1 (1360 BS), 13-15.

SANATHANA e HAZARIKA 2020: Y.S. Sanathana e M. Hazarika, «Iconography of Sage Narada in Indian Sculptural Art Regional Variations», Ancient Asia 11/3 (2020), 1-11.

SB (Śatapathabrāhmaṇa): GRETIL e-text, inserimento del testo a cura di H.S. ANANTHANARAYANA e W.P. LEHMAN. Disponibile su: <http://gretil.sub.uni-goettingen.de/gretil/1_sanskr/1_veda/2_bra/ satapath/sb_14_u.htm> (ultimo accesso: 1 aprile 2021).

SEN 1914: D.C. Sen, Vanga Sahitya Parichaya or Selections from the Bengali Literature from the Earliest Times to the Middle of the Nineteenth Century, with Illustrations: Vol. I, Calcutta 1914.

SEN 1924: B.K. Sen, «Rāmāi Paṇdit», Calcutta Review, August issue (1924), 353-361.

ŚIB (Śibāyan): D.C. Bhațțācārya, Ā. Bhațțācārya (sam.), Śibāyan. Rām'krș̣na Kabicandra Racit, Kalikātā 1383 BS.

SMITH 1999: W.L. Smith, «Śiva, Lord of the Plough», in R.P. Das (ed.), Essays on Middle Bengali Literature, Calcutta, 208-228.

ŚP (Śūnya Purānn): (1) N.N. Basu (sam.) (1314BS), Śūnyapurāṇ. Rāmāipaṇdit pranīt, Kalikātā, 1314 BS

(2) C.C. Bandyopādhyāỳ (sam.) Śūnyapurāṇ. Muhāmad Śahīdullāh o Basanta Kumār Catțopādhyā̄y 
likhite bhūmikā, Kalikātā 1336 BS. (3) B.M. Cațțopādhyāỷ (sam.), Rāmāipaṇdit biracit Śūnyapurāṇ (Śūnyapurāṇ, Sañgajāt-paddhati, Dharmapurāṇ), Kalikātā 1977.

ŚS (Śib'samkīrttan): Y. Hāldār (sam.), Rāmeśbarer Śib-Saṃkīrttan bā Śibāyaan, Kalikātā 1957.

STEWART 2019: T.K. Stewart, Witness to Marvels: Sufism and Literary Imagination. Islamic humanities, Oakland, 2019.

TS (Taittirīyasaṃhitā): FUSHIMI, M., ed., 2002, TITUS (Thesaurus Indogermanischer Text- und Sprachmaterialien) Text collection, Black Yajur-Veda, Taittirìya-Saṃhitā, sulla base dell'edizione curate da Albrecht WEBER, Die Taittirîya-Saṃhitâ, Leipzig: Brockhaus, 1871-1872 (Indische Studien, 11-12), TITUS version by Jost Gippert, Frankfurt. Disponibile su: <http://titus.uni-frankfurt.de/ texte/etcs/ind/aind/ved/yvs/ts/ts.htm> (ultimo accesso: 1 aprile 2021)

TURNER 1962-1966: R.L. Turner, A Comparative Dictionary of Indo-Aryan Languages, London 1962-1966.

VĀ (Vrkșāyurveda): R.P. Das, Das Wissen von der Lebensspanne der Bäume. Surapālas Vṛssāyurveda kritisch ediert, übers und kommentiert von Rahul Peter Das; mit einem Nachtrag von G. Jan Meulenbeld zu seinem Verzeichnis "Sanskrit names of plants and their botanical equivalents", Stuttgart 1988.

VALENTIJN 1724: F. Valentijn, Oud en nieuw Oost-Indiën: vervattende een naaukeurige en uitvoerige verhandelinge van Nederlands mogentheyd in die gewesten, benevens eene wydluftige beschryvinge der Moluccos, Amboina, Banda, Timor, en Solor, Java, en alle de eylanden onder dezelve landbestieringen behoorende : het Nederlands comptoir op Suratte, en de levens der Groote Mogols: als ook een keurylke verhandeling van 't wezentlykste dat men behoort te weten van Choromandel, Pegu, Arracan, Bengale, Mocha, Persien, Malacca, Sumatra, Ceylon, Malabar, Celebes of Macassar, China, Japan, Tayouan of Formosa, Tonkin, Cambodia, Siam, Borneo, Bali, Kaap der Goede Hoop en van Mauritius: te zamen dus behelzende niet alleen eene zeer nette beschryving van alles, wat Nederlands Oost-Indiën betreft, maar ook 't voornaamste dat eenigzins tot eenige andere Europeërs, in die Gewesten, betrekking heeft, Amsterdam 1724 .

WITZEL 2009: M. Witzel, «The linguistic history of some Indian domestic plants», Journal of Biosciences, 34/6 (2009), 829-833.

WojTILLA 1991: G. Wojtilla, «The Krșiśāsana. The Manual of agricultural implements in Sanskrit: A description of the ploughs types», Tools and Tillage, 6/4 (1991), 202-209.

WojTILLA 2006: G. Wojtilla, History of Krșiśāstra: A History of Indian Literature on Traditional Agriculture, Beiträge Zur Kenntnis Südasiatischer Sprachen Und Literaturen 14, Wiesbaden, 2006.

\section{NOTE}

1. Già nell'Atharvaveda compaiono numerosi riferimenti al riso (AVŚ VI.140.2ab; VIII.2.18ab, VIII. 7.20cd; IX.1.22cd, IX.6.14ab; X.6.24cd; XI.4.13ab, XI.4.13cd; XII.1.42ab). Nello Yajurveda nero troviamo inoltre menzionate le saptá grāmyoșadhayah, le «sette piante domestiche» (riso, orzo, sesamo, fagiolo indiano verde, miglio, frumento e lenticchie) (TS v.2.5.5), che - con l'aggiunta di panìco, fagiolo mungo e fagiolo di Madras - diventeranno dieci (SB XIV.9.3.22).

2. MANU II.22ab.

3. Il testo, in pracrito di Aśoka e alfabeto brāhmī, fa riferimento alla distribuzione di un surplus di riso (dhānya) verso le popolazioni limitrofe a causa di una carestia (EI XXI, 83-91). Sull'origine dei centri di produzione del cibo in India, si veda: WITZEL 2009, 829. 
4. Sulla nascita e lo sviluppo di una cultura del riso in India, si vedano i contributi raccolti in GOPAL e SRIVASTAVA 2008 e LUDDEN 1999. Sulla letteratura agronomica, wOJTILLA 2006. Sull'agricoltura in Bengala, DAS GUPTA 1929.

5. Per semplicità, i nomi degli dèi saranno trascritti adottando il sistema IAST e non le regole di traslitterazione dal bengali. Avremo quindi Śiva, Pārvatī, Viśvakarma anziché Śib, Pārbatī, Biśbakarma.

6. Le fonti in bengali qui usate ricorrono spesso alla datazione interna. In tal caso, l'anno di pubblicazione è seguito dalla sigla BS (bānglā san). Per calcolare l'equivalente anno gregoriano bisogna aggiungere 593 o 594 anni, a seconda che la data sia prima o dopo pahelā baiśākh o nababarșa, il Capodanno bengalese (14/15 aprile).

7. BHAKTIBINODE 1313 BS; DASGUPTA 1995, 399-412; SEN 1924. Sul Śūnyapurāṇ, rimandiamo a BASU, 1316 BS; RĀY, 1326 BS e 1338 BS. Si vedano anche le introduzioni in ŚP ${ }_{1}$, ŚP ${ }_{2}$ e ŚP ${ }_{3}$ a cura rispettivamente di N.N. Basu; M. Śahīdullāh e B.K. Cațțopādhyāẏ; e B.M. Caț̣topādhyāày.

8. Desumiamo ciò dalla struttura prosodica del testo. I metri in medio-bengali usavano avere un numero di piedi (mâtrā) corrispondente a quello delle sillabe. A partire dal xV secolo, si registra un cambiamento fonetico: la vocale breve /a/ non è pronunciata a fine parola dopo una consonante isolata (e.g. /deba/ [debo] > [deb]) e cade per effetto dell'accento posto sulla prima sillaba della parola seguente. Pertanto, il numero di sillabe diminuisce e non corrisponde più al numero di piedi.

9. Il paȳār è costituito da due emistichi, il primo di otto piedi (mātrā) e il secondo di sei. Il tripadī, che può essere laghu tripadī $(6+6+8 m \bar{a} t r \bar{a})$ o dïrgha tripadī $(8+8+10 m \bar{a} t r \bar{a})$, presenta due cesure e una rima interna.

10. Si veda la sezione Śiber gān pariśișța in SEN 1914, 156-161.

11. Il devoto che prende parte alla Carak pūjā è generalmente descritto mentre compie una rotazione. Tale pratica, oggi molto diffusa, rappresenta un'innovazione in cui prevale l'elemento ludico. Si veda per una disamina accurata FERRARI 2010, 145-151.

12. Sul Gājan, si veda MITRA 1972; FERRARI 2010.

13. CM p. 102 (Śib'pujā pracār). La traslitterazione dal bengali riflette i materiali manoscritti e le fonti primarie consultate e pertanto può non corrispondere ad un'ortografia standard. Si veda al proposito STEWART 2019, xxv-Xxx.

14. Parte della sezione successiva è stata trattata, seppur in modo meno approfondito, in FERRARI 2016.

15. ŚP ${ }_{1}$ (pp. 107-115): 80 versi in paȳär; ŚP ${ }_{3}$ (pp. 234-243 = DHPB pp. 227-237): 122 versi non numerati, principalmente in paȳār.

16. Lo stile scarno e il linguaggio che troviamo in ŚP, ricco di inflessioni dialettali, ne fa un documento di grande importanza rispetto allo stile più artificioso ed elegante degli Śibāyan (BHAȚṬĀCĀRYA 2000; BHAȚṬĀCĀRYA 1951, 199-200; CLARK 1955, 504-506; SMITH 1999).

17. In ŚP, così come nella letteratura Śibāẏan, la figura di Śiva non è da intendersi come quella del rinunciante o dell'asceta itinerante codificata nella letteratura normativa sanscrita. Si tratta a tutti gli effetti di un mendicante il cui aspetto sudicio insieme al suo comportamento antisociale viene spesso enfatizzato, soprattutto nelle rappresentazioni del teatro popolare bengalese $(\dot{y} \bar{a} t r \bar{a})$. 18. ŚP ${ }_{1}$ p. 108.

19. Si tratta dei frutti di haritakì (Terminalia chebula RETZ.), una pianta nota anche come śivā, e di beherāa (= vibhïtaka) (Terminalia bellirica (GAERTN.) ROXB.).

20. ŚP ${ }_{1}$ p. 108.

21. ŚP ${ }_{1}$ p. 109.

22. La «quintuplice ambrosia»: un'offerta di yoghurt, latte, burro chiarificato, miele e zucchero.

23. ŚS 2015-2226.

24. ŚS 2108-2100. 
25. La figura di Hanumān, divinità marziale principalmente associata al culto di Rāma, è invocata nella letteratura scientifica sanscrita per la distruzione di agenti infestanti dei campi (VĀ XV.162; KP Xxx.195).

26. Il calendario agricolo può variare tra regione e regione. Di seguito, la progressione delle attività nei campi in relazioni ai vari mesi nell'area bengalese: (1) Baiśākh (aprile/maggio): fertilizzazione dei campi, prima aratura, (prima) semina a secco; (2) Jyaiștha (maggio/giugno): seconda fertilizzazione dei campi, essicazione dei semi, seconda aratura; (3) Āṣāṛh (giugno/ luglio): fertilizzazione dei campi, aratura e lavorazione dei campi durante l'allagamento, semina durante l'allagamento, crescita e trapianto delle plantule, mondatura dei campi, aratura dopo la semina; (4) Śrābạ̣ (luglio/agosto): fertilizzazione dei campi, trapianto delle plantule, estirpazione delle erbacce, aratura dopo la semina, disposizione delle plantule; (5) Bhādra ( agosto- settembre): ulteriore trapianto, controllo dei livelli dell'acqua e di eventuali parassiti e erbe infestanti, primo raccolto; (6) Āśbin (settembre/ottobre): controllo dei livelli dell'acqua e di eventuali parassiti e erbe infestanti, fertilizzazione, ultima aratura, raccolto; (7) Kārttik (ottobre/ novembre): raccolto; (8) Agrahāẏan (novembre/dicembre): raccolto, trebbiatura, allagamento dei campi (se necessario); (9) Pouṣ (dicembre/gennaio): ultimo raccolto, trebbiatura; (10) Māgh (gennaio/febbraio): stasi e preparazione del terreno; (11-12): Phālgun-Caitra ( febbraio/aprile): fertilizzazione e aratura. Per quanto riguarda il numero di raccolti, le fonti medievali divergono in modo significativo. Risulta quindi difficile poter identificare quali qualità di riso producevano raccolti singoli, doppi, ecc., anche in virtù del fatto che manca una tassonomia ufficiale di riferimento.

27. ŚP ${ }_{1}$ pp. 109-110.

28. Śiva dai «tre occhi».

29. La tabella è derivata da un nostro studio precedente (F ERRARI 2016, 259). Senza una descrizione morfologica risulta estremamente difficile identificare le varietà di riso menzionate in ŚP 110-115. La maggior parte di esse è stata ricostruita sulla base degli elenchi forniti dalle seguenti fonti: RRI 1980; CRRI 2012; DRD 2013. Le varietà indicate con * non sono state identificate.

30. LEWIN 1912, 233. Cfr. HJ 460.

31. ŚP ${ }_{3}$ p. 239.

32. hâla: «aratro». Si riferisce al tratto di terreno che un singolo aratro è capace di lavorare in un giorno.

33. Cfr. ŚP ${ }_{1}$ 112.45: suniā krodhita hoila har mahāsae / sunu bhìm khetti se dhāne āguni bhejāe //

34. ŚP 1 p. 239.

35. LEWIN 1869, 11

36. dubbār gāngeta bahutakhāni joli («molti stretti canali dal Gange tumultuoso») (ŚP ${ }_{3} 111$, v. 44a).

37. Una versione ridotta e priva di traduzione del presente studio sul ḍheńkī si trova in FERRARI 2016, 260-263.

38. In questo articolo, utilizzeremo la grafia dhếkī perché ricorrente nei testi da noi consultati. Tuttavia, lo stesso termine si può trovare nelle forme ḍ̂êki, ḍheniki o ḍheniki dal momento che la pronuncia in bengali non cambia. Tale lessema non è direttamente riconducibile ad alcun termine sanscrito, potrebbe essere una parola onomatopeica derivata dal suono (dhak dhak) prodotto dal pestello quando va a colpire il mortaio (BŚK 1008). Forme lessicali simili sono attestate in svariate lingue MIA e NIA e vanno sempre ad indicare una leva che permette di schiacciare, frantumare o spremere (e.g. cereali, semi, pietre, ecc.) (GRIERSON 1926, 90, 118, 206; TURNER 1962, 317).

39. Per un uso alternativo del dhễkī quale la manifattura della carta, si veda PASTENA 2017, 191.

40. Mangifera indica L. e Artocarpus heterophyllus LAM. 
41. Il processo di mondatura prosegue con l'uso del ventilabro (śūrpa), un attrezzo che, a differenza del ḍhêkī, è menzionato nei manuali di agricoltura in sanscrito (KKS 568-569).

42. L'unico Krișiśāstra a menzionare un attrezzo simile al ḍhêkī è il Krșiśāsana (x.79) di Daśaratha, un lavoro molto tardo composto nel 1909, dove però è chiamato dhauka (WoJTILLA 1991).

43. āvedanakena vinā utkrișțī na grāhyā / vākpārușyadandapārușyayoh sākșitve sārī na grāhyā / ḍhenkukaḍ̂hakaniladumphakāśca viștịn na kārayitavyāḥ [*] prapāpū[ra]kagopālāh rājagrahāna na grāhyā $\left[h^{*}\right]$ / grhā- (prima piastra, ln. 8) (SIRCAR 1953-1954, 172). Si veda anche dhemikā in DNM IV. 17.

44. FERRARI 2015, 56.

45. Musici celesti.

46. Il testo fa riferimento a Setāi Paṇdit, Nīlāi Paṇụit, Kamasāi Paṇḍit e Rāmāi Paṇdit che, secondo lo schema cosmologico dello Śūnyapurān, svolgono la loro attività rispettivamente durante Satya Yuga, Tretā Yuga, Dvāpara Yuga e Kali Yuga. Un quinto specialista rituale, Gosāi Paṇdit, è invece invocato con riferimento all'ultima epoca, lo Śūnya Yuga.

47. Si tratta di Candra, Hanumān, Sūrya e Garuḍa, che presidiano rispettivamente Satya Yuga, Tretā Yuga, Dvāpara Yuga e Kali Yuga. Il gufo Ullūka, veicolo di Dharma Ṭhākur, è invece il guardiano dello Śūnya Yuga.

48. Il volo di Nārada è altrimenti descritto in ŚS (pp. 240-41, vv. 2334-55, metro: paȳār). Si veda anche, il terzo atto di Matsyadharā Nāṭak, una breve commedia scritta da Kālidās Mukhopādhyāỳ e pubblicata tra il 1873/74, dove il ḍhêkī e Nārada discutono sull'iniziativa di Śiva di dedicarsi all'agricoltura (MUKHOPĀDHYĀỲ 1280 BS, 22-38).

49. Le fonti più antiche sono il Dharmamangal di Rūp'rām Cakrabartī (1584 Śaka = 1662 d.C.) e lo Anādimañgal di Rām'dās Ādak (1584 Śaka = 1662 d.c.). Il Dharmapurān di Maȳūr'bhātța, menzionato sia da Rūp'rām che da Rām'dās, e quindi ad essi precedente, è andato perduto (ŚAHĪDULLĀH 1360 BS; BHAṬṬĀCĀRYA 1959). Si noti che tutti gli autori dei Dharmamañgal'kābya provengono da villaggi siti in Rāạh (CURLEY 2008, 148-149).

50. La presenza di Rāmāi non è confermata in tutti i Dharmamañgal. In questo genere, si tratta infatti di un personaggio minore (BHAṬṬĀCĀRYA 2000, 722-23).

51. «Sospensione sull'albero di śal (Shorea robusta C.F.GAERTN.) (DHM ${ }^{\mathrm{Gh}} 33, \mathrm{~V} .21 ; \mathrm{DHM}^{\mathrm{NB}}$ 53, VI.153).

52. L'eco della presenza di Haricandrarāja si trova in alcuni Dharmamangal nell'episodio detto Haricandrapālā, un'interpolazione narrata dalla stessa Rañjābatī, la quale fa riferimento alla storia di Hariścandra e della sua regina mentre vagano in una zona del Bengala ricca di fiumi allo scopo di ottenere la grazia di un figlio tramite pratiche ascetiche e di rinuncia. Si veda al proposito MANRING 2018, dove però è trattato solamente il testo di Rūp'rām Cakrabartī.

53. Sul ruolo di ritualista di corte di Rāmāi Paṇdit, si veda B HAKTIBINODE 1313 BS; SeN 1924; DASGUPTA 1995, 399-412.

54. BHATtASAli 1920. Cfr. DASGUPTA 1976, 464.

55. GRIERSON 1878, 9-11; BHATTTĀĀĀRYA 1961.

56. EI IX, 229-233. Si veda anche la Storia del Buddhismo in India (dPal dus kyi 'khor lo'i chos bskor gyi byung khungs nyer mkho) di Lama Tāranātha (1575-1634) (CHATTOPADHYAYA 1997, 104).

57. Si veda nota 52 supra.

58. Sulla letteratura di frontiera nel periodo premoderno in Bengala, rimandiamo a EATON 2017 e D'HUBERT 2018, 1-88.

59. RAỲ 1420 BS, 486-487.

60. Si veda la a piastra di rame di Tipperah (VII secolo) in cui ci si riferisce al distretto (vișaya) di Suvvunga (=Sylhet), una regione densa di foreste, con «spessi intrichi di vegetazione e rampicanti» (sambhogagahanagulmalatāvitāne) e dove non vi è distinzione tra "ciò che è naturale $\mathrm{e}$ ciò che è fatto dall'uomo» (krtākrta) (v. 22) (EI XV, 311). 
61. Il fiume Meghna, originariamente un canale del Vecchio Brahmaputra, si divide in Meghna superiore, che nasce dalla confluenza dei fiumi Surma e Kushiyara, e Meghna inferiore, che si va a formare quando ha ricevuto i fiumi Surma (Barak) e, più a sud, Padma e Jamuna (il nome del Brahmaputra in Bangladesh).

62. EI vol. VI, 207

63. ChaKRABARTY e MANDAL 2019, 545. Nel periodo medievale solo in Bengala meridionale e orientale conobbero importante sviluppo economico legato ad un surplus della produzione agricola (EATON 2000, 194-207).

64. FEDERICI 1587, 90.

65. AN III.160-161 (pp. 226-228). Cfr. il riferimento al corso del Gange nei pressi di Tāṇ̣̂a in ivi 291 (p. 428, n. 2).

66. RAY் 1420 BS, 72-81; EATON 2000, 194-207.

67. māgh māse gõsāi pithibi mañgalila / jataguli bhum prabhu sakali casila // 19

bhūme cās diā prabhu bhūma kaila tathā / bīc bhoj nahi duggā bala tār kathā // 20

68. bhāddar māseta haila dhān ati manuhar / dahar dāngar saba ekui susar // 27

āsin māseta meghe bārisae jhisikāni / nadie ācen kūp jal pūrita je pāni // 28

69. CATLING et al. 1983; KAR et al. 2012, 574.

70. CLARKe 1888, 287.

71. La dinastia Sena (1095-1230), originaria del Karnataka, è tradizionalmente considerata vaiṣnava, probabilmente in virtù del fatto che il grande poeta Jayadeva compose il Gittāgovinda sotto gli auspici di Lakșmaṇasena (1179-1207), il sovrano più importante della dinastia. In realtà, i Sena furono sostenitori dell'ordine tradizionale brahmanico e sostennero tutte le correnti religiose.

\section{RIASSUNTI}

In questo articolo, andremo ad analizzare il mito della nascita del riso (dhānyer janma) nei testi in medio bengali attribuiti a Rāmāi Paṇdit, il primo officiante del culto di Dharmarāj. Tale narrativa, che vede protagonista una forma di Śiva agreste (krșak debatā), è stata a lunga considerata una tarda interpolazione dovuta alla diffusione della cultura śivaita bengalese a scapito di culti ritenuti indigeni. La nostra analisi intende dimostrare come l'elemento śivaita sia originato nell'ambito del folklore nāth del Bengala orientale e sia precedente al culto di Dharmarāj. Al fine di dimostrare ciò, proponiamo una lettura incrociata dei materiali interni alla tradizione dharmaita, della letteratura nāth, di fonti epigrafiche e di una serie di rilievi storico-ambientali relativi allo slittamento idrogeologico dei complessi fluviali del Bengala. Lo studio, in ultima analisi, offre una chiave di lettura per meglio comprendere forme di mitopoiesi e dinamiche di circolazione di materiali narrativi e rituali nel Bengala medievale in relazione alle tecniche di coltivazione e alla biodiversità inerenti alla cultura cerealicola del tempo.

In this article, I will examine the myth of the origin of rice (dhänyer janma) in the texts attributed to Rāmāi Paṇdit, the first priest of the Bengali god Dharmarāj. The myth, which features a form of Śiva as a ploughing god (krșak debatā), has long been considered a late interpolation following the spread of the Bengali śaiva culture. In fact, the śaiva (nāth) element is earlier than Dharmarāj mythology and, with all probability, originated in eastern Bengal. This thesis will be discussed 
along a cross-examination Dharmaite literature, Bengali Nāth texts, epigraphic sources, and a historical study of the hydrogeological shift of the Bengal river complexes. In so doing, the paper offers a unique perspective to appreciate forms of mythopoesis and the dynamics of circulation of narratives and rituals in medieval Bengal with respect to agricultural knowledge and the biodiversity inherent to Bengali cereal culture.

\section{INDICE}

Parole chiave : Hinduismo, Bengala, riso, Gājan, Śiva

Keywords : Hinduism, Bengal, rice, Gājan, Śiva

\section{AUTORE}

\section{FABRIZIO FERRARI}

Dipartimento di Scienze Storiche, Geografiche e dell'Antichità (DiSSGeA)

Università degli Studi di Padova

Palazzo del Liviano

Piazza Capitaniato, 7

35139 Padova

fabrizio.ferrari.1(at)unipd.it 